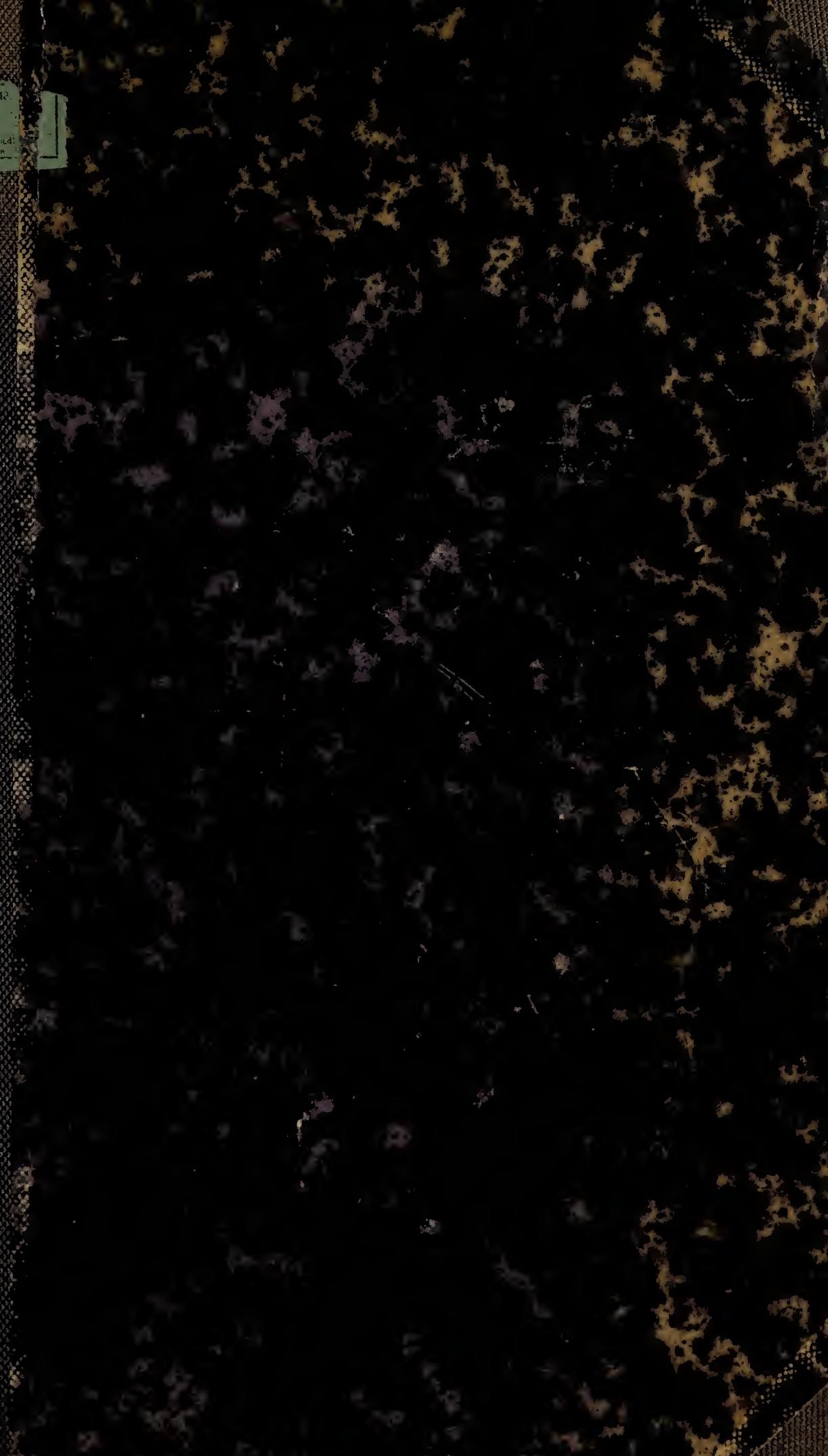



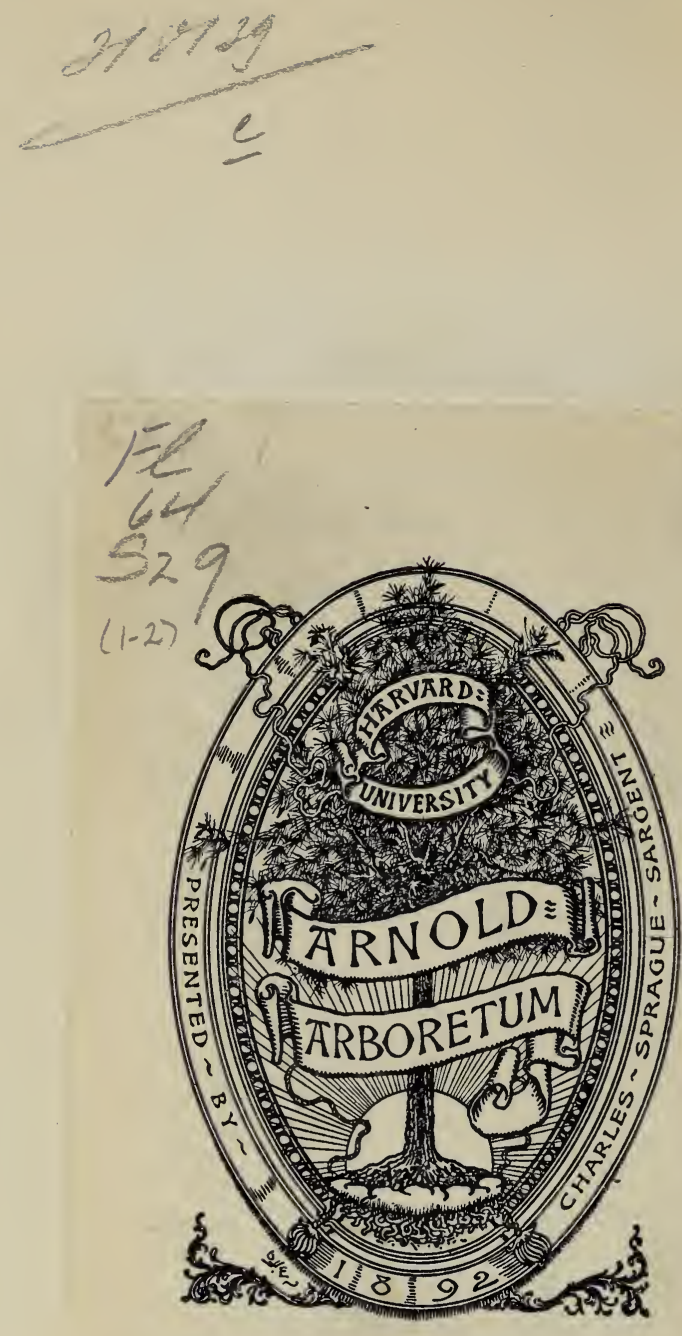

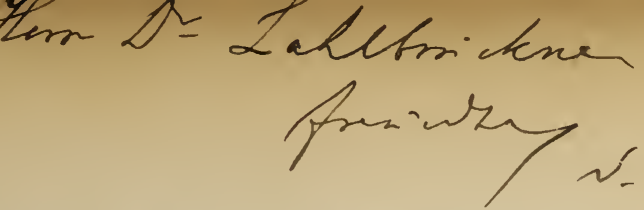

\section{POLYPETALAE DISGIFLORAE}

\section{REHMANNIANAE}

sive

\section{ENUMERATIO}

Linearum, Halpighiacearum, Zygophyllearum, Geraniacearum, Rutacearum, Ochnacearum, Burseracearum, Meliacearum, Olacinearum, Ilicinearum, Celastrinearum, Rhamnearum, Ampelidearum, Sapindacearum, Anacardiacearumque

a cl. Dr. A. Rehmaun

annis I875-I880 in Africa australi extratropica collectarum

Auctore

Dr. Ign. Szyszyłowicz.

CRACOVIAE.

TYPIS UNIVERSITATIS JAGELL ONICAE provisore A. M. Kosterkiewicz.

1888. 



\section{Polypetalae Disciflorae Rehmannianae}

sive enumeratio

Linearum, Malpighiacearum, Zygophyllearum, Geraniacearum, Rutacearum, Ochnacearum, Burseracearum, Meliacearum, Olacinearum, Ilicinearum, Celastrinearum, Rhamnearum, Ampelidearum, Sapindacearum, Anacardiacearumque

a Cl. Dr. A. Rehmann

annis $1875-1880$ in Africa australi extratropica collectarum

auctore

Dr. Ign. Szyszyłowicz.

\section{I N E A E.}

Linum thesioides Bartl., Linn., VII, pag. 540 sec. Harv. et Sond., Flor. Cap., I. pag. 310.

Eckl. et Zey. 270! Drege!

Promontorium Bonae Spei: Cape Town, Stinkwater 1381; Cape flats 2190.

Outeniqua Mnts.: Montagu Pass 238, 239.

Linum Thunbergii Eckl. et Zey. 275! excl. syn. Harv. et Sond., Flor. Cap., I. pag. 310.

Promontorium Bonae Spei: Rondebosh 1684.

Transvant: Boshveld Klippan 5204, Houtbosh 6342. 
Erythroxylon emarginatum Schum. et Thon., Guin. Pl., 224 sec. Oliver, Flor. of Trop. Afr. I., pag. 274; Erythroxylon caffrum Sond. in Linn. XXIII., pag. 22; Harv. et Sond., Flor. Cap. I., pag. 233.

Gueinzius 52! 587!

NATAL: Maritzburg 7551.

Erythroxylon pictum E. Mey. in herb. Drege! Harv. et Sond., Flor. Cap. I, pag. 234.

NATAL: Inanda (leg. J. Wood.); Umbilo ad catarrhactam 8115 .

\section{MALPIGHIACEAE.}

Acridocarpus natalitius Juss. Mon. pag. 232 sec. Harv. et Sond., Flor. Cap., I pag. 231, Banisteria Kraussiana Hoch.

Harv., Thes. Cap., I. tab. 19 !

Gueinzius Natal 392!

NataL: Vernlam 9064; Inanda (leg. J. Wood).

Sphedamnocarpus galphimiaefolius Juss. sub Acridocarpo Mon. pag. 273 sec. Harv. et Sond., Flor. Cap., I, pag. 232. Gueinzius! sub Banisteria leavigata Sond.

Transvaal: Boshveld Pinaasriver 4790.

Sphedamnocarpus pruriens Juss., sub Acridocarpo Mon. pag. 238 sec. Harv. et Sond., Flor. Cap. I., pag. 232. Drege! sub Banisteria.

Natal: Durban 8770, Inanda (leg. Wood.).

TransvaAL: Makapansberge Streydpoort 5493.

forma major nov, form.

forma omnis partibus robustior.

Transvaal: Pretoria Aapies Poort 4194. 


\section{Sphedamnocarpus Rehmanii nov. sp.}

S. fruticosus, caule ramoso, volubili, ramis teretibus flavido-sericeis, foliis $10-30 \mathrm{~mm}$. lge. petiolatis, laminibus ovatis, basi subcordatis, subtus biglandulosis, apice acuminatis, integris, utrinque pilosis, subtus glaucis, ad nervos prominentes flavido-sericeis, $35-80 \mathrm{~mm}$. lgis., $30-45 \mathrm{~mm}$. ltis., flores umbellati- $3-4$ flori, terminales, pedunculis $10-13 \mathrm{~mm}$. lgis., pedicellis $18-20 \mathrm{~mm}$. lgis., flavido-sericeis, sepalis ovatis, pubescentibus, $4-5$ $\mathrm{mm}$. lgis,, petalis obovatis sepalis longioribus, carpella 3 , axi coalita, birsuta, styli 3 , filiformes, divergentes; fructus samarae 3 , oblongae, superne in alam productae, flavido-sericeae.

Sphedamnocarpo galphimiaefolio Juss. affinis indole eglandulororum foliorum bene distinguenda.

Transvaal: Houtbosh 6390.

\section{Triaspis Rehmannii nov. sp.}

T. fruticosa, ramis teretibus, erectis, glauco-tomentosis, foliis subsessilibus vel $1-2 \mathrm{~mm}$. lge. petiolatis, oppositis, laminibus ovatis, basi rotundatis subcordatisve, apice acuminatis, abrupte acuteque cuspidatis, integris, glaucis, supra sparse pilosis, subtus ad nervos prominentes tomentosis, $15-20 \mathrm{~mm}$. lgis., $10-12 \mathrm{~mm}$. ltis.; flores racemosi, axillares, pedunculis $10-12 \mathrm{~mm}$. lgis., pedicellis $10-20 \mathrm{~mm}$. lgis., tomentosis, sepalis lanceolato-ovatis, obtusis, tomentosis $3-4 \mathrm{~mm}$. lgis., petalis unguiculatis, inaequalibus, uno margine longe fimbriato, alteris subintegris, $10-12 \mathrm{~mm}$. lgis., filamentis inaequalibus, glabris, ovario piloso; samarae 3, margine 8-10 $\mathrm{mm}$. late alatae. 
Triaspidi macropteronti Welw. proxima differt magnitudine foliorum glabritudineqne filamentorum.

Transvanu: Pretoria Aapies Poort 4195; Boshveld Elandsriver and drift 4944.

\section{ZYGOPHYLLEAE.}

Tribulus terrestris Linn., Sp. plant. p. 554 sec. Harv. et Sond., Flor. Cap. I., pag. 352, 353.

Eckl. et Zey. 751!

Cape Town: 3336.

Griqualand W.: Kimberlay 3442.

Transvaal: Boshveld, Klippan 5207.

var, hispidissimus Sond. in Harv. et Sond., Flor. Cap. l. c.

Transvaau: Boshveld Kameelpoort 4826, Elandsriver Neu Halle 4907.

Damaraland: (leg. . . . ).

var. desertorum Sond. in Harv. et Sond., Flor. Cap. l. c.

Eckl. et Zey. 751. $\beta$ !

Oranje Fr. St.: Mudriverdrift 3586.

Koup: Flakkraal 2982.

Augea capensis Thunb. Flor. cap. pag. 389; Harv. et Sond., Flor. Cap. I. pag. 355.

In planitie carroidea prope Gwaakriver ad viam publicam inter Gray Reims et Aberdenn (leg. Bolus 528).

Zygophyllum sessilifolium Linn., Sp. plant., pag. 522. sec. Harv. et Sond., Flor. Cap. I, pag. 358. 
Eckl. et Zey. 761! sub Zygophyllo limoso, Eckl. et Zey. 765 ! pr. prt. sub Zygophyllo Commelini.

Promontorium Bonae Spei: Cape town, Cape flats $2180,2181$.

Zygophyllum flexuosum Eokl. et Zey. 768 sec. Harv. et Sond., Flor. Cap., I., pag. 359.

var. cuneatum Szysz.

var. $\beta$. foliis cuneato-oblongis Sond. in Harv. et Sond. Flor. Cap. I., pag. 359.

Drege 7166 !

Drakenstein Berge. Tulbagh 2234 a.

Ceres Distr.: Michelspass $2334 \mathrm{~b}$.

Zygophyllum divaricatum Eckl. et Zey. 766! Harv. et Sond., Flor. Cap. I., pag. 360.

Prope Algou Bay: (leg. Bolus 2665).

Zygophyllum Morgsana Linn., Sp. plant., pag. 551. sec. Harv. et Sond., Flor. Cap. I., pag. 360.

Drege 7162 ! 7163 !

KnysNa DISTR.: Belveder 4508.

Zygophyllum microphyllum Linn. f. Suppl. pag. 232 sec. Harv. et Sond., Flor. Cap., I., pag. 362.

RogGEFELD: Beaufort W. 3132.

Griqualand W.: Eitalers Fontein 3355.

Zygophyllum glaucum E. Mey. in Harv. et Sond., Flor. Cap., I., pag. 362 .

ROGGEFELD: Beaufort W. 3156.

Zygophyllum microcarpum Lichtenst. herb. Cham. et Scblecht. Linn. V, rag. 45 sec. Harv. et Sond., Flor. Cap., I, pag. 363. 
In carroideis prope Great Reinet alt. 2500' (leg. Mac. Owan 1760).

\section{GERANIACEAE.}

Monsonia ovata Cav. Diss. tab. 113. fig. 1!; Harv. et Sond., Flor. Cap. I. pag. 255.

Eckl. et Zey. 439! 440! Monsonia praemorsa E. Mey. in Drege!

Promontorium Bonae Spei: (leg. J. Cooper).

Outeniqua Mnts.: Montagu Pass 253.

KNYSNA DISTR: Belveder 461.

var. biflora Harv. in Harv. et Sond., Flor. Cap. I. pag. 255. Monsonia biflora Eckl. et Zey. 441!

NATAL: Durban 8871.

Transvaal: Pretoria, colles supra Aapiesriver 4349, Wonderboompoort 4573, Houtbosh 6323.

var. lancifolia nov, var.

foliis angustis, cuneato-lanceolatis.

TransvaAl: Makapansberge Streydpoort 5498.

Monsonia biflora DC., Prodrm. I, pag. 638; Harv. et Sond., Flor. Cap. I., pag. 255; Oliver Flor. of Trop. Afr., I, pag. 290.

Monsonia angustifolia E. Mey. in Drege!

Oranje Fr. St.: Draaifontein 3671; Bloemfontein 3869. Transvaal: Hogge Veld Bronkersprint 6571; Boshveld inter Elandsriver et Klippan 5016.

Monsonia Burkeana Planch. in herb. Hook. sec. Harv. et Sond., Flor. Cap., I, pag. 255. 
Transvaal: Houtbosh 6322.

Oranje Fr. St.: Olifantsfontein 3511.

Monsonia attenuata Harv. in Harv. et Sond., Flor. Cap., I, pag. 255.

Natal: Westtown Mooiriver 7351; Drakensberg Goldstream 6915.

Transvaal: Hogge Veld Standarton 6819.

Monsonia speciosa Linn. f. Suppl. pag. 342 sec. Harv. et Sond., Flor. Cap., I, pag. 256.

Bot. Mag. tab. 73! Cav. Diss., tab. 74, fig. 1 !

Eckl. et Zey. 444! Drege!

Promontorium Bonae Spei: in monte Diaboli 1133.

Oranje Fr. St.: Bloemfontein 3855.

Geranium incanum Linn., Sp. plant., pag. 957 sec. Harv. et Sond., Flor. Cap., I., pag. 257.

Eckl. et Zey. 445! Drege 7510 a! d!

Pronontorium Bonae Spei: Cape Town, Cape flats 2152, Outeniqua Mnts.: Montagu Pass 252.

Geranium sericeum Harv. in Harv. et Sond., Flor. Cap., I, pag. 257.

- Geranium incanum E. Mey. in Drege!

Oranje Fr. St.: Witteberge, Kadziherg 3992.

Geranium canescens L'Her. tab. 38 sec. Harv. et Sond., Flor. Cap., I, pag. 257.

Drege 750

Outeniqua Mnts.: Montagu Pass 256.

Geranium ornithopodum Eckl. et Zey, sec. Harv. et Sond., Flor. Cap. I, pag. 250.

Drege 7513 a!, Burchell Cat. 6037! 
NATAL: Drakensberg, Goldstream 6935; Durban 8873; Inanda (leg. J. Wood).

Erodium moschatum Willd., Sp. pl. IV. pag. 631; Harv. et Sond., Flor. Cap., I, pag. 259.

Engl. Bot. 905!

Promonturium Bonae Spei: Cape Town, Stinkwater 1368.

Pelargonium longifolium Jacq., Jc. Rar. tab. 518 sec. Harv. et Sond., Flor. Cap., I, pag. 261.

var. ciliatum Harv. in Harv. et Sond., Flor. Cap. I, pag. 262. Knysna Distr.: Belveder 478.

\section{Pelargonium Tysonii Szysz.}

Geranium ciliatum Cav. Diss. pag. 234, Tab. 118 fig. 2 !

In clivis montosis prope Murraysburg alt. $4100^{\prime}$ (leg. W. Tyson. no. 328).

\section{Pelargonium Rehmannii nov. sp.}

P. acaule, totum cano-tomentosum, radice tuberoso, foliis $140-230 \mathrm{~mm}$. lge. petiolatis, radicalibus, laminibus ovatis, basi truncatis vel dilatate incisis, apice rotundatis subacuminatisve, margine obtuseincisis inaequaliterque acute crenatis, utrinque dense cano-holosericeis, 60-100 mm. lgis., 70-90 mm. ltis.; stipulis lanceolatis, tomentosis, $20-30 \mathrm{~mm}$. lgis.; pedunculus simplex, subteres, cano-tomentosus, 35-40 cm. lg.; bracteae multae lanceolatoovatae, tomentosae; flores 25-30 in umbellam compositi, pedicellis $20-40 \mathrm{~mm}$. Igis., calyx cano tomentosus, eglandulosus, sepalis lanceolatis, 8-10 mm. lgis., tubo $5-7$ ns. brevioribus, corolla 5-pe- 
tala, petalis subaequalibus ovatis, basi cuneatis, 12-15 mm. lgis.

Planta superba Pelargoniae Grenvilleae Andr. proxima sed optime distinguenda.

Transvaal: Boshveld Menaarsfarm 4852.

Pelargonium barbatum Jacq., Jc. rar., tab. 513 sec. Harv. et Sond., Flor. Cap, I, pag. 267.

Drege!

Promontorium Bonae Spei: in monte Diaboli 1128.

Pelargonium astragalifolium Pers., Ench., II, pag. 227 sec. Harv. et Sond., Flor. Cap., I. pag. 268.

var. minor Harv, in Harv. et Sond., Flor. Cap. I, pag. 268. Pelargonium pinnatum Andr. Eckl. J. U. 604!

Promontorium Bonae Spei: in monte Diaboli 1126 p. p.

var. foliosum Harv. in Harv. et Sond., Flor. Cap. I, pag. 268.

Eckl. et Zey. 496! sub Hoarea; Drege 7499!

Promontorium Bonae Spei : in monte Diaboli 1126 p. p.

Pelargonium rapaceum Jacq,, Jc. rar., tab. 510! Harv. et Sond., Flor. Cap. I, pag. 269.

CEREs distr.: Michelspass 2345, 2346.

Pelargonium lobatum Willd., Sp. plant., III, pag. 650 sec. Harv. et Sond, Flor. Cap., I, pag. 272.

Cav. Diss. tab. 114!

Eckl. et Zey. 507! sub Polyactio sphondylifolio; Drege!

KNysna Distr.: Belveder 462; Portland 367.

Pelargonium pulverulentum Colv. in Sw. Ger., tab. 218 sec. Harv. et Sond., Flor. Cap. I, pag. 272, 273. 
var. pedicellatum Harv. in Harv. et Sond., Flor. Cap., I, pag. 273.

Natal: Inanda (leg. J. Wood).

Pelargonium triste Ait., Hort. Kew., I, vol. 2, pag. 418 sec. Harv. et Sond., Flor. Cap., I, pag. 274.

var. daucifolium Harv. in Harv. et Sond., Flor. Cap., I, pag. 274.

Eckl. et Zey. 513! sub Polyactio triste; 521! sub Polyactio daucifolio; 511! sub Polyactio filipendulifolio; E. Mey. sub Pelargonio filipendulifolio in Drege!

Promontorium Bonae Spei: Cape Town, Cape flats 2149.

var. filipendulifolium Harv. in Harv. et Sond., Flor. Cap. I, pag. 274.

Pelargonium triste E. Mey. in Drege!

Promontorium Bonae Spei: Cape Town, Cape flats 2150.

Pelargonium aconitifolium Eckl. et Zey. 517!; Harv. et Sond., Flor. Cap., I, pag. 276.

Pelargoniurn polymorphum E. Mey. in Drege!

NataL: Oakfort, Umblotiriver 8515.

Transvaal: Houtbosh 6324, 6325.

Pelargonium Zeyheri Harv. in Harv. et Sond., Flor. Cap. I, pag. $626,627$.

Transvaal: Boshveld, Menaarsfarm 4851.

Pelargonium flabellifolium Harv, in Harv. et Sond., Flor. Cap. I, pag. 277.

Transvaal: Houtbosh 6326.

Natal: Inanda 8398. 
Pelargonium ceratophyllum L'Her., Ger., tab. 13 sec. Harv. et Sond., Flor. Cap., I, pag. 279.

Bot. Mag. 315!

Worcester Distr.: Hexrivervaley 2823, Groote Tafelberg 2776.

Pelargonium dissectum Eckl. et Zey., 536!; Harv. et Sond., Flor. Cap., I, pag. 281.

Clanvilliam (leg. P. A. Mader).

Pelargonium abrotanifolium Jacq., Hort. Schoenbr., tab. 136 sec. Harv. et Sond., Flor. Cap. I, pag. 282.

Murraysburg: (leg. W. Tyson 231).

Pelargonium ramosissimum Willd., Spec. plant., III, pag. 688 sec. Harv. et Sond., Flor. Cap. I, pag. 283.

Murraysiburg: (leg. W. Tyson 267).

Pelargonium myrrhifolium Ait., Kew., I, vol. 2, pag. 421 sec. Harv. et Sond., Flor. Cap. I, pag. 286.

var. fruticosum Harv. in Harv. et Sond., Flor. Cap. I, pag. 286. Eckl. et Zey. 557!

Promontorium Bonae Spei: Cape Town, Cape flats 2151.

var. longicaule Harv., in Harv. et Sond., Flor. Cap. I, pag. 287.

Eckl. et Zey. 550! sub Myrrhidio.

Promontorium Bonae Spei: Cape Town, Cape flats 2148 , Stinkwater 1366 ; Rondebosh 1691; in monte Diaboli 1131.

var. lacerum Harv. in Harv. et Sond., Flor. Cap. I, pag. 287. Eckl. et Zey. 555! sub Myrrhidio.

Promontorium Bonae Spei: Cape Town, Stinkwater 1367. 
Pelargonium multicaule Jacq., Ic. rar., tab. 534 sec. Harv. et Sond., Flor. Cap. I, pag. 287.

Eckl. et Zey. 5522! sub Myrrhidio; Eckl. et Zey. 553! sub Myrrhidio triangulari; Drege 7442, a!, 7487 !

Transvaal: Houtbosh 6327.

District of Queenstown: (leg. Cooper 433).

Pelargonium grossularioides Ait., Hort. Kew., I, vol. 2, pag. 42 sec. Harv. et Sond., Flor. Cap. I, pag. 289.

var. anceps. Harv. in Harv. et Sond., Flor. Cap. I, pag. 289. Eckl. et Zey. 562! 561!; Drege 746!

Promontorium Boxae Spei: Cape Town 1545.

IN MONTIBUS WORCESTER: 2853.

Boschberg: (leg. Mac Owan 114).

Albert Distr.: (T. Cooper 685̃ sub Pelargonio multi. caule T. Cooper non Jacq.!)

KNYSNA DISTR.: Belveder 464.

Natal: Durban 8872.

Outeniqua Mrts.: Montagu Pass. $25 \check{5}$.

Pelargonium fumarioides L'Her, in Herb. Banks. sec. Harv. et Sond. Flor. Cap. I. pag. 290.

Pelargonium columbinum E. Mey. in Drege!

AlberT DisTr.: (leg. T. Cooper 791).

Pelargonium ovale Burm., Cap., 19 sec. Harv, et Sond., Flor. Cap. I, pag. 291.

Car., Diss., pag. 238, tab. 103, fig. 3!

Hexriverberge: Axellsfarm 2729.

Pelargonium peltatum Ait.., Hort. Kew., II, pag. 427 sec. Harv. et Sond. Flor. Cap. I. pag. 293.

Drege 7459 ! 
var. clypeatum Harv. in Harv. et Sond., Flor. Cap. I, pag. 294.

Near the source of Kuskamma: (leg. T. Cooper 436 sub Pelargonio grossularioide Cooper non. Ait.!)

Pelargonium saniculaefolium Willd, Sp. plant., 3, pag. 673 sec. Harv. et Sond., F'lor. Cap. I.pag. 294. Geranium tabulare Cav. Dis. tab. 100, fig. 2! Eckl. et Zey. 603! sub Eumorpha nobili; Drege $7455 ! 7456$ !

Promontorium Bonae Spei: in monte Diaboli 1130.

Pelargonium patulum Jacq., Ic. rar., tab. 541 sec. Harv. et Sond., Flor. Cap., I. pag. 295.

var. tenuilobum Harv. in Harv. et Sond., Flor. Cap. I, pag. 295.

Eckl. et Zey. 607! sub Eumorpha tenuiloba; Pelargoniuin patulum E. Mey. in Drege!

Ceres distr.: Michelspass 2343.

Pelargonium alchemilloides Willd., Sp. plant., III, pag. 656 sec. Harv. et Sond., Flor. Cap., I. pag. 295. Cav. Diss., tab. 98, fig. 1 !

var. dentatum Harv, in Harv. et Sond," Flor. Cap. I., pag. $295,296$.

Eckl. et Zey. 592! sub Isopetalo; Drege 7462, a! Outeniqua Mnts.: Montagu Pass 254. NataL: Inanda 8400; Drakensberg, Biggarsberge 7103. Craddock distr.: (leg. T. Cooper 1293).

var. aphanoides Harv. in Harv. et Sond., Flor. Cap., I. pag. 296.

Kaffraria: (leg. T. Cooper 429, 431). 
Pelargonium glaucum L'Her., Ger., tab. 29 sec. Harv. et Sond., Flor. Cap. I, pag. 296.

Geranium lanceolatum Cav., Diss., tab. 102, fig. 2 ! Bot. Mag. 56!

In montibus supra Worcester 2452.

Pelargonium acetosum Ait., Hort. Kew., II, pag. 430 sec. Harv. et Sond. Flor. Cap. I. pag. 298.

Craddock Distr.: (leg. T. Cooper 495).

Pelargonium zonale Willd., Sp. plant., III, pag. 667 sec. Harv. et Sond. Flor. Cap. I, pag. 298.

Albany: (leg. T. Cooper 15666).

Pelargonium betulinum Ait., Hort. Kew., II, pag. 429 sec. Harv. et Sond., Flor. Cap. I. pag. 301.

Bot. Mag. 148!

Drege 7451 !

Promontorium Bonae Spei: Cape Town, Cape flats 2146.

Pelargonium cucullatum Ait., Hork. Kew., II, pag. 426 sec. Harv. et Sond., Flor. Cap. I, pag. 302.

Cav., Diss., tab. 105. fig. 1 !

Eckl. et Zey. 621!

Promontorium Bonae Spei: in monte Diaboli 1125.

Pelargonium angulosum Ait., Hort., Kew., II, pag. 426 sec. Harv. et Soud., Flor. Cap. I, pag. 302.

var. truncatum Szysz.

Pelorgonium angulosum var angulosum Harv. in Harv. et Sond., Flor. Cap., I, pag. 302.

Eckl. et Zey. 625! Drege 7448!

Outeniqua Mrs.: Montagu Pass. 257. 
Pelargonium capitatum Ait., Hort. Kew., II, pag. 425 sec Harv. et Sond., Flor. Cap. I, pag. 303.

Cav., Diss., 105, fig. 1!

Eckl. et Zey, 630! Drege!

Pronontorium Bonae Spei: Cape Town, Cape flats 2147; Campsbay 1603.

Pelargonium vitifolium Ait., Hort. Kew., II, pag. 425 sec. Harv. et Sond., Flor. Cap. I, pag. 303.

Cav. Diss., 111, fig. 2 !

Eckl. et Zey. 631! Drege!

Knysna DISTR.: Belveder 463.

NATAL: Inanda 8399.

Pelargonium crispum Ait., Hort. Kew., II, pag. 430 sec. Harv. et Sond. Flor. Cap. I, pag. 304.

Cav., Diss., tab. 109, fig. 2 !

Eckl. et Zey. 635!

WorCester: Brandcley 2380.

Pelargonium scabrum Ait., Hort. Kew., II, pag. 430 sec.

Harv. et Sond., Flor. Cap., I, pag. 304.

Cav., Diss, tab. 108, fig. 1 !

Drege 7445 !

in montibus supra Worcester 2451.

Ceres Distr.: Michelspass 2347.

Pelargonium hispidum Willd., Sp. plant., III, pag. 677 sec.

Harv. et Sond., Flor. Cap., I. pag. 307.

Cav. Diss., tab. 110, fig. 1 !

Eckl. et Zey. 616! Drege!

in montibus supra Worcester 2454.

Pelargonium Radula Ait., Hort. Kew., II, pag. 423 sec. Harv. et Sond., Flor. Cap. I. pag. 307. 
Eckl. et Zey. 644!; Eckl. et Zey. 645! sub $P_{e}$ largonio roseo; Drege 7444!

Transvaal: Hogge Veld, Standarton nr.?

Oxalis monophylla Linn., Syst. plant., pag. 432 sec. Harv. et Sond. Flor. Cap. I. pag. 318.

var. stenophylla Sond. in Harv. et Sond., Flor. Cap. I, pag. 318.

Oxalis stenophylla Meissn. in Krauss!

in pascuis PROPE Tulbagh (leg. Krauss).

Oxalis glabra Thunb., Diss., 17, fig. 2 sec. Harv. et Sond. Flor. Cap. I, pag. 319 .

var. pusilla Sond., in Harv. et Sond., Flor. Cap., I. pag. 320. Oxalis pusilla Jacq. Ox., tab. 42 !

Eckl. et Zey., 692!

Promontorium Bonae Sper: Cape Town, Cape flats $2173,2174$.

Oxalis versicolor Linn., Sp. plant. pag. 622 sec. Harv. et Sond. Flor. Cap. I, pag. 321.

Jacq. Oxal. 36 !; 77, fig. 4 !

Promontorium Bonae Spei: Cape flats 1497 b, 1502, 2178.

Oxalis polyphylla Jacq., Oxal., tab. 39!; Harv. et Sond., Flor. Cap. I, pag. 321.

Promentorium Bonae Spei: Cape Town, Cape flats $2171,2172$.

var. filifolia Sond., in Harv et Sond. Flor. Cap. I., pag. 321. Oxalis revoluta E. Mey. in Drege! 
Promontorium Bonae Spei: Cape Town 1496; in monte Tabulari $838,840$.

Oxalis tenuifolia Jacq., Oxal., tab. 38!; Harv. et Sond., Flor. Cap. I. pag. 323.

Eckl. et Zey. 683! 684! 685!

Promontorium Bonae Spei: Cape Town, 1491; Rondebosh 1695, 1696.

Oxalis purpurea Thunb., Cap. 535 excl. syn. Jacq. sec. Harv. et Sond., Flor. Cap. I, pag. 331.

Eckl. et Zey. 707 !, 706 !

foliis cuneatis, corolla anguste longeque tubulosa.

Promontorium Bonae Spei: Cape Town 1511, 1512, 1513; Stinkwater 1330; in monte Diaboli 1140.

Oxalis variabilis Lind1., Bot. Beg., tab. 1505! Harv. et Sond., Flor. Cap. I, pag. 331.

var. nana Sond. in Harv. et Sond., Flor. Cap., I, pag. 331. Drege 2117!, Oxalis speciosa v. purpurea E. Mey. in Drege!

Promontorium Bonae Spei: Cape Town 1510.

Oxalis pulchella Jacq., Oxal., tab. 69!; Harv. et Sond. Flor Cap. I, pag. 332.

var. glabrata Sond., in Harv. et Sond., Flor. Cap. I, pag. 332. NATAL: Maritzburg 7592.

Oxalis commutata Sond. in Harv. et Sond., Flor. Cap. I, pag. 333.

Oxalis tenella Eckl. et Zey. 728!; Oxalis erubescens E. Mey. in Drege!

Promontorium Bonae Spei: Cape Town, Cape flats 2177. 
Transvaal: Houtbosh 6391.

ORANJE Fr. St.: Bloemfontein 3876.

Oxalis punctata Linn., f. pag. 243 sec. Harv. et Sond., Flor. Cap., I, pag. 334.

Eckl. et Zey. 730 !; 731 ! sub Oxalide calcaria.

Promontorium Bonae Spei: 'Cape Town 1506, 1507.

Oxalis luteola Jacq., Oxal., tab. 65!; Harv. et Sond., Flor. Cap. I, pag. 335.

Eckl. et Zey. 718 !; 717 !

Transvaal: Houtbosh 6394.

\section{Oxalis Mariae Szysz.}

Oxalis rigidula Eckl. et Zey. 709 ! pr. prt.; Oxalis strumosa E. Mey. in Drege (?); Oxalis lutea V. marginata Sond. in Harv. et Sond., Flor. Cap. I, pag. 335. (?)

Foliola subsessilia, obverse triangularia, truncata vel subrotundata, margine distante obtuse crenulata, utrinque glabra, nervo marginibusque subhirtis; scapi uniflori, bracteolis linearibus $4-5 \mathrm{~mm}$. lgis.; corolla calice duplo longior, lutea externe violaceo-emarginata.

Oxalidi luteo proxima differt forma foliorum, longitudine indoleque bracteolarum et colore corollae.

TransvaAL: Pretoria Wonderboomport 4571.

Oxalis imbricata Eckl. et Zev. 736!; Harv. et Sond, Flor. Cap. I, pag. 337.

var. rosea Sond. in Harv. et Sond., Flor. Cap. I, pag. 337. Somerset EAst.: (leg. Mac. Owan 1694). 
Oxalis obtusa Jacq., Oxal, tab. 79, fig. 1! ; Harv. et Sond., Flor. Cap. I, pag. 337.

Eckl. et Zey. 732!; 735 sub Oxalide cuprea Lodd. Promontorium Bonae Spei : Cape Town, Cape flats 2179, Stiukwater 1333.

Oxalis lanata Linn. f. Suppl., pag. 244 exc. syn. sec. Harv. et Sond., Flor. Cap., I, pag. 338.

Jacq., Oxal., tab. 77, fig. 2 !

Eckl. et Zey. 721! Eckl. U. J. 593! sub O. livida,

Promontorium Bonae Sper: Cape Town, 1495, 1505. Stinkwater 1331.

Oxalis bifida Thunb., Diss., 16, fig. 1 sec. Harv. et Sond., Flor. Cap., I, pag. 341.

Jacq., Oxal., tab. 79, fig. 4 !

Eckl. et Zey. 664!

Promontorium Bonae Spei: Cape Town 1499, 1500, 1501, Stinkwater 1335 a, 1336, in monte Tabulari 839.

Oxalis bifurca Lodd., Bot. Cab., tab. 1056 sec. Harv. et Sond., Flor. Cap. I, pag. 342.

Promontorium Bonae Sper: Cape Town, Stinkwater, $1335 \mathrm{~b}$; Rondebosh 1697.

var. incana Harv. in Harv. et Sond. Flor. Cap. I, pag. 342. Eckl. et Zey. 669! pro sp.

Promontorium Bunae Spei: in monte Diaboli 1139; Campsbay 1592; Cape Town 1498, Stinkwater 1337.

Oxalis Smithii Sond., in Harv. et Sond., Flor. Cap. I, pag. 342 , 
var. latifolia Sond. in Harv. et Sond., Flor. Cap. I, pa. 34ะ. Oxalis bisulca E. Mey. in Drege!

TransvaAL: Houtbosh 6392.

Oxalis hirta Linn., Sp. plant., pag. 623 sec. Harv. et Sond., Flor. Cap. I, pag. 343.

Jacq. Oxal. tab. 13 !; tab. 14 ! sub 0 . hirtella; tab. 15 ! sub 0 . multiflora; tab. 16 ! sub O. rubella; tab. 10 ! sub O. tubiflora.

Eckl. J. U., 594!; Eckl. et Zey. 673!, 676 !, 677 !, 678 !, 679 !

Promontorium Bonae Spei: Cape Town 1487, 1488, 1489 , 1490; Campsbay 1590, 1591; Stinkwater 1332; Rondebosh 1698, 1699, (3386 leg. Bolus).

Oxalis tomentosa Linn., Syst., pag. 434 sec. Harv. et Sond. Flor. Cap. 1, pag. 347. Jacq., Oxal., tab. 81 !

Promontorium Bonae Spei: Cape Town 1486.

Oxalis flava Linn., Sp. plant. pag. 621 sec. Harv. et Sond., Flor. Cap. I, pag. 347.

var. Thunbergiana Sond. in Harv. et Sond., Flor. Cap. I, pag. 347. Jacq., Oxal., tab. 73 ! 78 fig. 2.

Promontorium Bonae Spei : Cape Town, 1485, 1497a; Campsbay 1593.

Oxalis sericea Linn. f., Suppl., pag. 243 sec. Harv. et Sond., Flor. Cap. I, pag. 348.

Jacq., Oxal., tab. 77, fig. 1 !

Eckl. et Zey. 658!; Eckl. J. U. 592 !

Promontorium Bonae Spei: Cape Town 1493, 1494; Campsbay 1589. 
Oxalis cernua Thunb., Diss., no. 12, tab. 2 sec. Harv. et Sond., Flor. Cap. I, pag. 348.

Jacq., Oxal., tab. 6!

Eckl. et Zey. 657!

Promontorium Bonae Sper: Cape Town 1492, Cape flats 2176.

Oxalis caprina Lin., Syst., pag. 433 sec. Harv. et Sond., Flor. Cap. I, pag. 349.

Eckl. et Zey. 655! sub O. macrophylla Horn.; Eckl. et Zey. 656 ! sub 0 . dentata.

Transvaal: Houtbosh 6393.

Oxalis livida Jacq., Oxal., tab. 8!; Harv. et Sond. Flor. Cap. I, pag. 349.

Eckl. et Zey. 654!

Promontorium Bonae Spei: Cape Town 1508.

Oxalis semiloba Sond. in Harv. et Sond., Flor. Cap. I. pag. 350.

NATAL: Durban 8773; Inanda (leg. Wood).

Oxalis corniculata Linn., Sp. plant., 624 sec. Harv. et Sond., Flor. C'ap. I, pag. 351; Jacq., Oxal., tab. 5!

Eckl. et Zey. 648!; Oxalis ceratilis E. Mey. in Drege!

Promontorium Bonae Spei: Cape Town 1509, Stinkwater 1334.

Oranje Fr. St.: Bloemfontein 3841 !

Outeniqua mnts: Montagu Pass 251.

form. uniflora nov. form.

Natal: Maritzburg 7593.

Impatiens capensis Thunb., Prodrm., pag. 41 ; Harv. et Sond., Flor. Cap. I, pag. 312. 
Natal: Camperdown 7784; Drakensberg Mountainprospect 6962.

Transvaal: Houtbosh 6399, 6400.

George Town Distr.: Oakfort 555.

\section{RUTACEAE.}

Calodendron capense Thunb., Nov. Gen. P., 2, pag. 41 sec. Harv. et Sond. Flor. Cap. I, pag. 371.

Eckl. et Zey. 778! Drege! Mac Owan 16135!

NATAL: Inanda (leg. Wood).

Kaffraria : (leg. Cooper 84).

Macrostylis villosa Sond. in Harv. et Sond., Flor. Cap. I, pag. 439.

Eckl. et Zey. 817 ! sub M. lanceolata; Drege!

var. glabrata Sond. in Harv. et Sond., Flor. Cap. I, pag. 439.

Promontorium Bonae Spei: Cape Town, Cape flats 2217.

In clivis montis Muizenberg (leg. H. Bolus 45̃25).

Diosma succulenta Berg., Pl. Cap., pag. 63 exc. syn. sec.

Harv. et Sond. Flor. Cap. I, pag. 373.

Drege! Scholl! Krauss!

Promontorium Bonae Spei: Cape Town, Cape flats 2211, Klapmoth; in monte Tabulari 807.

var. Lamarciziana Sond., in Harv. et Sond. Flor., Cap. I, pag. 373,374 .

Eckl. et Zey. 838, sub Diosma scabra Lam.

Promontorium Bonae Sper: Cape Town, Stinkwater 1341 ; in monte Diaboli $1151,1152$.

Diosma vulgaris Schl. Linn., VI, pag. 201 sec. Harv. et Sond., Flor. Cap. I, pag. 374. 
Promontorium Bonae Spei: Cape Town, Cape flats 2212, 2216; in monte Diaboli 1156, 1157.

var. longifolia Sond. in Harv. et Sond., Flor. Cap. I, pag. 375. Bot. Mag., 2332!

Promontorium Bonae Spei: Cape Town, Stinkwater 1344.

Drakenstein Berge: Bainskloof 2317.

Diosma virgata Mey., Spic. fl. Cap. sec. Harv. et Sond., Flor. Cap. I, pag. 375.

Eckl: et Zey. 848!

Promontorium Bonae Spei: Cape Town, Stinkwater 1339.

Coleonema pulchrum Hook., Bot. Mag. 3340! Harv et Sond., Flor. Cap. I, pag. 379.

Eckl. et Zey. 836 !; 832 sub C. virgato; 833 ! sub C. gracili.

In montibus Zumbergensibus: (leg. Leonard in hrb. Mac Owan).

Adenandra cuspidata Meyer. sec. Harv. et Sond., Flor. Cap. I, pag. 386.

var. glabra Sond., in Harv. et Sond., Flor. Cap. I, pag. 387.

Promontorium Bonae Spei: Cape Town, Cape flats 2213.

Sir Lawry's Pass: (leg. J. Spilhans).

var. villosa Sond. in Harv. et Sond., Flor. Cap. I, pag. 387. Promontorium Bonae Sper: Cape Town, Stinkwater 1342. 
Adenandra umbellata Willd., Enum. hort. Ber. pag. 257 sec. Harv. et Sond., Flor. Cap. I, pag. 387.

var. glandulosa B. et W. sec. Harv. et Sond., Flor. Cap. I, pag. 387.

Promontorium Bonae Spei: Cape Town, Stinkwater 1345.

Adenandra uniflora Willd., Enum. hort. Ber., pag. 256 sec. Harv. et Sond., Flor. Cap. I, pag. 388.

Eckl. et Zey. 793 !

Pronontorium Bonae Spei: Cape Town, Stinkwater 1343; Rondebosh 1678.

Adenandra marginata $R$. et Sch. sec. Harv. et Sond., Flor. Cap. I, pag. 391.

In collibus ditionis Riversdale: (leg. A. T. Hewitt in herb. Bolus 3688).

Adenandra humilis Eckl. et Zey. 784!; Harv. et Sond., Flor. Cap. I, pag. 392.

var. glabra Sond. in Harv. et Sond., Flor. Cap. I, pag. 392.

In montis Winterhocksberg Tulbaghensis: (leg. H. Bolus 5138).

Barosma pulchella B. et W. sec. Harv. et Sond., Flor. Cap., I, pag. 394.

Eckl. et Zey. 806!

IN SAXOSIS PRAERUPTIS MONTIS WINTERHOCKSBERG TULBaghensis (leg. H. Bolus 5139).

Barosma ovata B. et W. sec. Harv. et Sond., Flor. Cap. I, pag. 395 . 
var. vera Szysz.

Sond. sub var.. .) in Harv. et Sond., Flor. Cap., I, pag. 395.

Eckl. et Zey. 805!

Outeniqua masts.: Montagu pass 147.

var. cuneata Szysz.

Sond. sub var. o) in Harv. et Sond., Flor. Cap.

I, pag. 396.

Eckl. et Zey. 804! sub Barosma graveolente.

HeXriverberge: Axellsfarm 2717.

Barosma lanceolata Sond. in Harv. et Sond., Flor. Cap. I, pag. 397.

var. natalensis Sond. in Harv. et Sond., Flor. Cap. I, pag. 397.

Barosma Kraussiana Buching! in Krauss.

Natal: Inanda (leg. J. Wood 136).

Borosma scoparia Eckl. et Zey. 809!; Harv. et Sond., Flor. Cap. I, pag. 396.

KafFraria : (leg. T. Cooper 222).

Distr. of Albany: (leg. T. Cooper 107).

Distr. of Albert: (leg. T. Cooper 633).

Agathosma hirta Bart. et Wend. sec. Harv. et Sond., Flor. Cap. I, pag. 404.

Eckl. et Zey. 861! sub Agathosma biophylla.

V.an Stademberg (leg. Mac Owan 1035).

Agathosma umbellata Sond., in Harv. et Sond., Flor. Cap. I, pag. 408.

Eckl. et Zey. 855! sub Aguthosma bifida.

In clivis montosis inter Villiersdorp et French Hock. (leg. H. Bolus 5141).

Agathosma imbricata Willd., enum. Berol. pag. 259 sec. Harv. et Sond., Flor. Cap. I, pag. 418. 
var. reflexa Sond. in Harv. et Sond., Flor. Cap. I, pag. 418. Promontokium Bonae Spei: Cape Town, Stinkwater 1340.

var. acuminata Sond. in Harv. et Sond., Flor. Cap. I, pag. 419.

Promontorium Bonae Spei: in monte Diaboli 1154, 1155.

Agathosma rugosa Link., Enum. pag. 238 sec. Harv. et Sond., Flor. Cap. I, pag. 421.

var. glabra nov. var.

foliis utrinque glaberrimis.

Promontorium Bonae Spei: ad pedem montis Tabularis (leg. H. Bolus 4791).

var. lancifolia Szysz.

$\operatorname{var} \beta$ ) in Sond. et Harv., Flor. Cap. I, pag. 421. Promontorium Bonae Spei: Cape Town, Cape flats 2219 .

var. pubescens Szysz.

var. $\varepsilon$ ) in Sond. et Harv., Flor. Cap. I, pag. 421.

Promontorium Bonae Spei: Cape Town, Cape flats 2215

Agathosma marifolia Eckl. et Zey. 887!; Harv. et Sond., Flor. Cap. I, pag. 422 .

var. lanceolata Sond. in Harv. et Sond., Flor. Cap. I, pag. 422. Outeniqua mints.: Montagu Pass 145.

Agathosma cerefolia Bart. et Wend., Beitr. I, pag. 159; Harv. et Sond. Flur., Cap. I, pag. 424. 
var. glabrata Sond. in Harv. et Sond., Flor. Cap. I, pag. 424. Knysna DISTR.: Belveder 404.

Outeniqua mats.: Montagu Pass. 143.

Agathosma ciliata Link., Enum., I, pag. 238 sec. Harv. et Sond., Flor. Cap. I, pag. 425.

Eckl. et Zey., 878!

Promontorium Bonae Spei: in monte Diaboli (leg. H. Bolus 3940).

Agathosma ambigua Sond. in Harv. et Sond., Flor. Cap. I, pag. 426.

Eckl. et Zey. 895! sub Agathosma Thunbergiana.

In valle fluminis Palmiet: (leg. H. Bolus 5143).

Agathosma cuspidata Bart. et Wond. sec. Harv. et Sond., Flor. Cap., I, pag. 432.

var. glabra Sond. in Harv. et Sond., Flor. Cap. I, pag. 432. Eckl. et Zey. 900! sub Agathosma patentissima.

In convale prope cataractam Tulbaghensem (H. Bolus 5142 sub Agathosma commutata Bolus! non Sond.).

Agathosma chortophila Eokl. et Zey. 914!; Harv. et Sond., Flor. Cap. I, pag. 435.

In clivis montosis inter Villiersdorf et French Stock (leg H. Bolus 5140).

Empleurum serrulatum Ait., Hort. Kew., ed I, vl. 3, pag. 340 sec. Harv. et Sond., Flor. Cap. I, pag. 442.

Outeniqua masts.: Montagu Pass 141.

Zanthoxylum capense Harv. in Harv. et Sond., Flor. Cap. I, pag. 446. Tagaba capensis Thnb. Eckl, et Zey. 921! 
Natal: Durban 9046, 9047, 9048; in ditionibus occidentalibus (leg. H. Bolus 5144).

Transvaal: Houtbosh 6497; Pretoria Kuduspoort 4660, Aapies Poort 4094.

Zanthoxylum Thunbergii DC., Prodrm. I, pag. 726; Harv. et Sond., Flor. Cap. I, pag. 446.

Natal: Durban 9045? (ster.).

Transvaal: Houtbosh 6498? (ster.).

Outeniqua mnts.: Montagu Pass 211 ? (ster.).

Toddalia lanceolata A. Juss. sub Vepride sec. Harv. et Sond., Flor. Cap. I, pag. 447.

In simvis Boschberg Somerset: (leg. Mac Owan 410)

Clausena inaequalis Benth., Flor. Nigr., pag. 257; Oliver, Flor. of Trop. Afr. I, pag. 307; Myaris inaequalis Presl., Bot. Bem. p. 40; Harv. et Sond., Flor. Cap. I, pag. 444; Clausena anisata Oliv. ? in Jour. of Linn. Soc. V, Suppl. 34.

Knysna Distr.: Esterneck 487.

Outeniqua mats: Montagu Pass 212.

Natal: Maritzburg 7494; Durban 9044; Drakensherg

Tugelariver Colenso 7165̃, Mountainprospect 6963 ;

Inanda (leg. Wood).

Transvaal: Houtbosh 6500, 6501.

\section{OCHNACEAE.}

Ochna Rehmannii nov. sp.

O. fruticosa, glabra, foliis alternis $3-4 \mathrm{~mm}$. lge. petiolatis, laminibus ovatis, utrinque acutis, minute serrato-ciliato-spinulosis, membranaceis, nervo primario secundariisque utrinque promi- 
minentibus, 50-60 mm. lgis, 25-32 mm. Itis; racemi axillares vel subterminales, simnlices, multiflori, subpenduli, 40-50 mm. lgi, in parte superiori ramulorum anni praeteriti aggregati; flores lutei (albi?), pedunculis simplicibus, unifloris, $8-10 \mathrm{~mm}$. lgis, sepalis 5, obtusis, petalis 5, sepalis minoribus; stamina multa, filamentis persistentibus glabris, sepalis dimidio minoribus, antheris elongatis, filamentis subaequilongis vel brevioribus, apice biporosis, connectivo elongato apice glandulifero brevissime rostratis; ovarium profunde septemlobatum; styli connati, centrales, apice liberi, stigmatibus capitellatis ; fructus?

Ochnae pulchrae Hook. proxima, differt antheris apice rostratis, basi astrumosis.

Transvanl: Yretoria solles supra Aapiesriver 4341.

Ochna atropurpurea DC., Ann. Mus., XVII, pag. 398 sec. Harv. et Sond., Flor. Cap. I, pag. 448.

Eck. et Zey. 925 ! sub Diporidio atropurpure Wendl.; Drege!

Natal: Camperdown 7786; Inanda (leg. Wood.).

Kaffraria: (leg. T. Cooper 376).

Quennstown Distr : (leg. T. Cooper 373).

Bassuta Land: (leg. T. Cooper).

var. natalitia Harv. in Harv. et Sond., Flor. Cap. I, pag. 448. Jiporidium natalitium Meissn. in Krauss!

NATAL: Drakensberg Van Reenenspass 7243; Durbau 890õ; Inanda (leg. J. Wood).

Ochna arborea Burch. Cat, 4012; DC., Prodrm. I, pag. 736; Harv. et Sond., Flor. Cap. I pag. 449.

Eckl. et Zey. 925! sub Diporidio arboreo W'ondl. 
Transtaal: Pretoria Wonderboompoort 4582, Aapiespoort 4083.

Knysna Distr.: Portland 359.

\section{BURSERACEAE.}

Commiphora Rehmanni Engler Anac. in DC., Mon. Phan., IV, pag. 15.

Transvaal: Boshveld, Klippan 5324.

\section{MELIACEAE.}

Turraea cbtusifolia Hochst., in Flora, XXVII, pag. 296; C. de Cand. in DC., Mon. Phan., I, pag. 440; Harv. et Sond., Flor. Cap. I, pag. 245; Oliver, Flor. of Trop. Afr., I, pag. 331.

Bot Mag. 6267!

NATAL: Durban 8913; Umbilo ad catarrhactam 8143.

Melia Azederach Linn., Sp. Plant., ed. III, pag. 550 sec. C. de Cand., in DC., Mon. Phan., I, pag. 451; Harv. et Sond, Flor. Cap. I, pag. 245; OIiver, Flor. of Trop. Afi. I, pag. 332.

Bot. Reg. 643!

Eckl. It. Un. 509! Drege!

Promontorium Bonae Spei: Cape Town 1551.

Natal: Durban 9049.

Ekebergia Mayeri Presl., Bot. Bem., pag. 25 sec. C. de Cand., in DC. Mon. Phan., I, pag. 642 ; Harv. et Sond., Flor. Cap., I, pag. 246.

Trichilia Ekebergia E. Mey. in Drege!

Knysna DISTR.: Esternek 486.

NATAL: Durban 9050. 
Ekebergia capensis DC., Prodrm. I, pag. 623; C. de Cand. in DC., Mon. Phan., I, pag. 641; Harv. et Sond. Flor. Cap., I., pag. 247.

NATAL: Oakfort Umhlotiriver 8513.

\section{OLACINEAE.}

Ximenia caffra Sond., Linn., XXIII, pag. 21 sec. Harv. et Sond., Flor. Cap. I., pag. 235.

Zeyer 1847 !

Transvaal: Pretoria colles supra Aapiesriver 435̃4; Nakapansberge Streydpoort 5465.

Apodytes dimidiata E. Mey. in Drege!; Harv. et Sond., Flor. Cap.

I, pag .235; Oliver, Flor. of Trop. Afr. I, pag. 335.

Pterocelastrus oltusus Hochst. in Krauss!; Pterocelastrus macrostylus Hochst. in Krauss!

NATAL: Durban 8958.

Transvaal : Houtbosh 6463, 646כ̃.

Cassinopsis capensis Sond. in Harv. et Sond., Flor. Cap. I, pag. 474.

Burcb., Cat. geogr. 5411!

Crlastrus ovatus E. Mey. in Drege!; Cassine ilicifolia Hochst. in Krauss!

Outeniqua mats.: Montagu Pass 187.

Kaffraria : (leg. T. Cooper 375).

\section{ILICINEAE.}

Ilex capensis Sond. et Harv. Flor. Cap. I, pag. 473.

Bot. Mag. 1858!

In convalle Mitchellaspass: (leg. H. Bolus 5202). 


\section{CELASTRINEAE.}

Catha edulis Forsk. Flor. Aeg. Arab. 63 sec. Oliv. Flor. of Trop. Afr. I. pag. 365, Methyscophylluin glaucum Eckl. et Zey. Enum. pag. 152, Harv. et Sond., Flor. Cap. 1, pag. 464.

Rich. Flor. Abyss. I, pag. 134. tab. 30 ! sub Catha Forskalii.

In dumetis ad ripes Zwaartkei prope Queenstown (Mac Owan 2179).

Hartogia capensis Thunb. Diss. nov. pl. Gen. 5. pag. 30̃ sec. Harv. et Sond., Flor. Cap. I., pag. 464.

var. lanceolata Sond. in Harv. et Sond., Flor. Cap. I., pag. 464. Eckl. et Zey. 980! sub H. lanceolata, Drege 2206! Scholl!

IN MONTIBUS SUPRA WORCESTER: 254 !

var. multiflora Eckl. et Zey. 981 ! pro spe.

Promontorium Bonae Spei: in monte Diaboli (?) 971. Ceres Distr.: Michelspass 2340.

Drakenstein BG.: Bainskloof 2288.

Cassine capensis Linn. Mant. 220 sec. Harv. et Sond., Flor. Cap. I., pag. 466.

Promontorium Bonae Spei: Cape Town, Stinkwater 1267.

Cassine barbara Linn. Sp. plant. 385 sec. Harv. et Sond., Flor. Cap. I., pag. 466.

Promontorium Bonae Sper: Houtbay 1612.

Cassine scandens Eckl. et Zey. 989! Harv. et Sond., Flor. Cap. I., pag. 467. 
var. latifolia Sond. in Harv. et Sond., Flor. Cap. I., pag. 467. KNYSNa DISTR.: Portland 354.

Cassine Maurocenia Linn., Spec. plant., pag. 385; Maurocenia capensis Sond. in Harv. et Sond., Flor. Cap. I., pag. 465.

Eckl. et Zey. 984!

Hook. Icon. plant. 552!

Promontorium Bonae Spei: Cape Town, Stinkwater 1265 ; Houtbay 1609.

Lauridia reticulata Eckl. et Zey. 968!; Harv. et Sond., Flor. Cap. I., pag. 469.

Promontorium Bonae Spex: Houtbay 1610, 1611, 1618

Cape Town, Stinkwater 1264.

Outeniqua Mnts.: Montagu Pass 192.

Gymnosporia acuminata Linn. Sp. plant. sub Celastro; Harv. et Sond., Flor. Cap. I., pag. 454.

Eckl. et Zey. 927! 930! sub C. mucronato, Drege 6745a sub Jice.

Natal: Drakensberg Van Reenenspass 7272.

Transvaal: Houtbosh 6427, 6511, 6512, 6517.

Gymnosporia polycantha Sond. in Harv. et Sond., Flor. Cap. I., pag. 455, sub Celastro.

Transvaal: Pretoria Aapies Poort 4080.

Gymnosporia tenuispina Sond. in Harv. et Sond. Flor. Cap. I, pag. 456 sub Celastro.

Transvaal: Boshveld inter Elandsriver and Klippan 5005, Menaarsfarm 4869.

Gymnosporia Zeyheri Sond. in Harv. et Sond., Flor. Cap. I., pag. 456 sub Celastro. 
In montibus Graaff Reinet: (leg. Bolus 576).

Gymnosporia undata Thun. Prodrm. pag. 42 sub Celastro sec. Harv. et Sond., Flor. Cap. I., pag. 457.

Eckl. et Zey. 953! sub C. ilicino.

Natal: Drakensberg Van Reenenspass 7281.

\section{Gymnosporia Rehmanni nov. sp.}

G. inermis, ramulis subangulosis, foliis alternis, breviter petiolatis, ovatis, apice subacutis, margine renıte adpresso serratis, coriaceis, utrinque glabris, glaucis, $45-55 \mathrm{~mm} . \mathrm{lg}$., $30-35 \mathrm{~mm}$. lat., petiolis 3-4 mm. lg.; flores fasciculati, axillares, pedunculis $7-10 \mathrm{~mm}$. lg.; capsula obovata, trigona, trilocularis, $5 \mathrm{~mm}$. lg., loculis bispermis; semina arillo completa e basi erecta; testa coriacea.

Natal: Camperdown 7707.

Gymnosporia buxifolia Linn. Sp. plant. 285 sec. Harv. et S nnd. Flor. Cap. I., pag. 459 sub Celastro.

var. laxiflora Sond. in Harv. et Sond., Flor. Cap. I., pag. 459. Promontorium Bonae Spei: Rondebosh 1650. Outeniqua Mnts.: Montagu Pass 232.

var. venenata Sond. in Harv. et Sond., Flor. Cap. I., pag. 459. Eckl. et Zey. 952. Zeyer 2182!

Promontorium Bonae Spei : Houtbay 1620, Rondebosh 1651; Cape Town, Cape flats 2200. Transvaal: Pretoria Aapies Poort 4081.

var. genuina Sond. in Harv. et Sond., Flor. Cap. I., pag. 459. Transvaal: Makapansberge Streydpoort 5458, Boshveld inter Elandsriver et Klippan 5006. 


\section{Gymnosporia (?) Woodii nov. sp.}

G. spinosa, ramulis teretibus, spinis paucis axillaribus, rectis, $10-11 \mathrm{~mm}$. lg.; foliis breviter petiolatis, obovatis, margine serratis, coriaceis, utrinque glabris, subglaucis, $40-50 \mathrm{~mm}$. lg., $20-30 \mathrm{~mm}$. lat., petiolis $2-3 \mathrm{~mm}$. lg., flores axillares, fasciculati, pedunculis $3-4 \mathrm{~mm}$. lg., flavescentes (?), 4 -meri, calice 4-partito, sepalis $2-2.5 \mathrm{~mm}$. lg., petalis obovatis $3-4 \mathrm{~mm}$. lg., staminibus 4 disci margini insertis petalis multo brevioribus; discus late explanatus cum ovario confiuens; ovarium 3 -loculare, stylus brevis, stigmatibus 3 ; ovnla in basi loculi 2 , erecta; fructus?

Natal: Inanda (leg. J. Wood).

Gymnosporia nemorosa Eckl. et Zey. 938 ! sub Celastro Harv. et Sond., Flor. Cap. I, pag. 460.

Transvaat: Houtbosh 6514.

Gymnosporia huillensis Welw. mss. sub Celastro in Oliv. Flor. of Trop. Afr. I, pag. 364.

Transvaal: Houtbosh 6518.

Gymnosporia luteola Delile sub Celastro in Ann. des. sc. nat. Ser. II. 20, pag. $90 \mathrm{sec}$. Oliver Flor. of Trop. Afr. I., pag. 363.

Ferr. et Gal. Voy. Abyss. Atl. Bot. tab. 8!

Schimp. It. Abyss. 840! 1596! sub C. simuatodentato Hochst.

Transvaal: Houtbosh 6516.

Gymnosporia laurina Eckl. et Zey. 966! sub Scytoplyllo; Harv, et Sond., Flor. Cap. I., pag. 471. 
Promontoricm Bonae Spei: Cape Town. Stinkwater 1268, Cape flats 2206, 2207, 2208, Houtbay 1617.

Drakenstein Bg.: Welington 2270.

WORCESTER: montes supra Worcester 2540, Brandvley 2392.

Ceres Distr.: Michelspass 2341.

Putterlickia verrucosa Sond. in Harv. et Sond., Flor. Cap. I., pag. 453 sub Celastro.

Gueinzius !

Natal: Durban 8989, 8990, 8991, 8992, Intschanga 7883.

Putterlickia pyracantha Linn. Sp. 285 sub Celastro; Harv. et Sond., Flor. Cap. I., pag. 453.

Bot. Mag. 1167! sub Celastro.

Eckl. et Zey. 937 sub Celastro campestri; Drege!

Knysna Dis'rR.: Belveder 398.

Elaeodendron glaucus nov. sp.

E. spinosus, ramulis teretibus, spinis axillaribus, rigidis, rectis, $3-60 \mathrm{~mm}$. lg.; foliis breviter petiolatis, fasciculatis, obovatis, apice obtusis, basi longe angustatis, integerrimis, coriaceis, utrinque glabris, glaucis, $15 \cdot 20 \mathrm{~mm}$. lg., 3-4 mm. lat. petiolis 1-2 mm. lg., stipulis minutis, caducis; cymae axillares, $30-60 \mathrm{~mm}$. lg., ramosae, pedunculis primariis $20-25 \mathrm{~mm}$. lg.; flores albi (lutei?) brevi-pedicellati, bibracteolati, bracteolis lanceolatis, margine ciliatis, $0.5-1 \mathrm{~mm}$. lat., pedicellis gracilibus, 3-7 mm. lg., calice 5-partito, sepalis ovatolanceolatis, margine ciliatis, $1.5-2 \mathrm{~mm}$. lg., petalis obovatis, 5-6 mm. lg., staminibus 5, sub margine disci insertis, $3-4 \mathrm{~mm}$. lg., filamentis filiformibus, antheris subglobosis; discus cupularis, sinuato 5-lobatus, ovarium disco sessili, 3-gonum, 
3-loculare, stigma sessile simplex vel sub 3-4 lobatum; ovula bina, e basi loculi erecta; drupa ovoidea, putamine osseo, 1-locularis; semen?

KARRow: Grootfontein 3014.

Elaeodendron capense Eckl, et Zey. 979!; Harv. et Sond., Flor. Cap. I., pag. 168.

Bot. Mag. 3835!

Outeniqua masts.: Montagu Pass 198.

NAtal: Durban 8895, 8897, 8898, Umbilo ad catarrhactam 8105̃, 8106.

Elaeodendron (?) Rehmannii nov. sp.

E. ramulis teretibus pubescentibus, foliis alternis breviter petiolatis, ovatis, apice obtusis, margine remote-adpresso serratis, coriaceis, subtus, praecipue nervis dense pubescentibus, $20--30 \mathrm{~mm} . \mathrm{lg}$., $14-20$ $\mathrm{mm}$. lat., petiolis pubescentibus 1.5-2 mm. lg.; flores umbellati, umbellis sessilibus, pedunculis pubescentibus, $3-5 \mathrm{~mm}$. $\mathrm{lg}$., flores colore?, 5 meri, sepalis pubescentibus, margine atrepunctatis, $0.5-1$ mm. lg., petalis obovatis, $2-3 \mathrm{~mm}$. lg.; staminibus sub margine disci quinquelobi insertis; ovarium cum disco confluens quinqueloculare, loculis biovulatis, ovulis e basi erectis; stylus brevis, stigmate simplici; fructus?

Transvanl: Pretoria Aapies Poort 4099.

Elaeodendron confertifolium Tulasn, Ann. des Sc. nat., 1857, pag. 106 sub „Mystroxylon" E. et Z., sec. Harv. et Sond., Flor. Cap. I., pag. 469.

var. leptocarpum Sond. in Harv. et Sond., Flor. Cap. I., pag. 469. Drege!

Transvaal: Houtbosh 6459. 
Salacia Kraussii Hochst. in Flora XXVII, I., pag. 306; Harv. et Sond., Flor. Cap. I., pag. 230.

Krauss! Guenizıus !

NATAL: Inanda (leg. J. Wood).

\section{RHAMNEAE.}

Zizyphus helvola Sond. in Harv. et Sond., Flor. Cap. I., pag. 476.

Zeyer 310 !

Transvaal: Makapansberge Streydpoort 5479; Pretoria Aapies Poort 4078; Houtbosh 6457.

Zizyphus Spina-Christi Willd., DC. Prodrm., II, pag. 20 sec. Oliver, Flor. of Trop. Afr. I, pag. 380.

Griqualand W.: Hünernestkloof 3394.

Oranje Fr. St : Mudriverdrift 3573, 3595.

Zizyphus mucronata Willd. En. Hort. Berol., pag. 251 sec. Harv. et Sond., Flor. Cap. I., pag. 475

Transvaal: Hogge Veld Doukershoek 6523; Houtbo:h 6458; Boshveld Menaarsfarm 4879.

var. pubescens Sond. in Harv. et Sond., Flor. Cap. I., pag. 476. Transvaal: Pretoria colles supra Aapiesriver 4352. NATAL: Maritzburg 7514, 7531, 7545; Inanda (leg. J. Wood).

var. glabrata Sond. in Harv. et Sond., Flor. Cap. I., pag. 476.

TransvaAl: Pretoria Aapies Poort 4079, Wonderboompoort 4572.

Natal: inter Pintown et Umbilo 8048; Maritzburg 7513; Camperdown 7699, 7758, 7785. 
Rhamnus prinoides l'Herit., sert. angl., 6, tab. 9, sec. Harv. et Sond., Flor. Cap. I., pay. 477.

Outeniqua mats.: Montagu Pass 191.

Natal: Drakensberg Van Reenenspass 7268.

Transvaal: Pretoria Aapies Poort 4084.

Oranje Fr. St.: Witteberge ad Caledonriver 3947.

Georgetown distr: Oakfort 563.

Caffraria : (leg. J. Cooper 278).

ShILOH: (leg. Baur 798).

Scutia indica Brogn. in Ann. Sc. Nat. Ser. I, X, 363 sec. Hook., Flor. of Br. Ind., I, pag. 640; Scutia Com. mersoni, Brogn. in Ann. Sc. Nat., Ser. I, X, pag. 363; Harv. et Sond., Flor. Cap. I., pag. 477.

Promontorium Bonae Spei: Rondebosh 1663.

Natal : Durban 9000, 9002; Umgeni 9001; Villdsbill . Pinetown 8016; inter Pintown et Umbilo 8057.

Phylica stipularis Linn., Mant. 208 sec. Harv. et Sond, Flor. Cap. I., pag. 482, Herb. Norm. Austr.- Afr. 134!; Drege! Eckl et Zey. 1008! sub Trichocephalo.

Promontorium Bonae Sper: Cape Town, Cape flats 2021 ; in monte Tabulari 783 ; in montibus supra Worcester 2533.

Phylica buxifolia Linn, Spec. plant., 283 sec. Harv. et Sond. Flor. Cap. I., pag. 482.

Herb. Nırm. Austr.- Afr. 131!, Burchell 8237! Eckl. et Zey. 1047! sub Soulangia.

Promontonium Bonae Spei: in monte Tabulari 782; Cape Town, Cape flats 2018, 2019.

Phylica paniculata Willd. Spec., I, par. 1112 sec. Harv. et Soud., Flor. Cap. I., pag. $4 \triangleleft 2$. 
Mac Owan in herb. Hance 16121! Drege 2224 b! Eckl. et Zey. 1046! sub Soulangia.

NATAL: Umbilo ad catarrhactam 8170.

Phylica oleoides DC., Prodrm., II, pag. 36 sec. Harv. et Sond., Flor.Cap. I., pag. 483.

Herb. Norm. Äustr.- Afr. '207!

Ad pedes montium prope catarrhactam Tulbaghensem (leg. H. Bolus 5058).

Phylica rigidifolia Sond. in Harv. et Sond., Flor. Cap. I., pag. 484. Drege 6771!

Cederbergen: (leg. H. Bolus 5611).

Phylica lutescens Sond. in Harv. et Sond., Flor. Cap. I., pag. 484.

Mac. Owan in Hance 16531!; Eckl. et Zey. 1043 ! sub Soulangia.

Oudeberg: prope Graaff Reinet (leg. H. Bolus 594). Albany • (leg. Cooper 1546).

Phylica viliosa Thunb., Flor. Cap I, pag. 202 sec. Harv. et Sond., Flor. Cap. I., pag. 485.

Drege 6767!

Kakroo: Witteberge Maggisfontein 2944.

var. glabrata Sond. in Harv. et Sond., Flor. Cap. I., pag. 486. Eckl. et Zey. 1042! sub Soulangia pinea.

In ditione Graaff Reinet: (leg. H. Bolus 824 sub Plyylica pariculata Bolus! non Willd. v. scabra H. Bolus).

Phylica plumosa Thunb., Flor. Cap., I, pag. 487 sec. Harv. et Sond., Flor. Cap. I., pag. 487.

Promontorium Bonae Spei: Cape Town, Stinkwater 1245, Cap. flats 2021. 
Phylica excelsa Wendl., Collect., III, pag. 3, tab. 74 sec. Harv. et Sond., Flor. Cap. I., pag. 488.

var. laxa Sond. in Harv. et Sond., Flor. Cap. I, pag. 488. Drege 6775 !

Hexriverberge: Axellsfarm 2711 (pr. prt.).

var. stricta Sond. in Harv. et Sond., Flor. Cap. I., pag. 488. Eckl. et Zey. 1029! sub Phylica cylindrica.

Drakenstein Berge: Bainskloof 2296.

var. papillosa Sond. in Harv. et Sond., Flor. Cap. I., pag. 488 IN MONTIBUS SUPRA WORCESTER: 2534, 2535.

var. brevifolia Sond. in Harv. et Sond., Flor. Cap. I., pag. 488. Eckl. et Zey. 1032! sub Phylica fulva.

HeXriverberge: Axellsfarm 2711 (pr. prt.).

Phylica capitata Thunb., Prodrm., pag. 45 sec. Harv. et Sond., Flor. Cap. I., pag. 490.

Herb. Norm. Austr.- Afr. 132 !, Burchell 8472 !

Promontorium Bonae Spei: Cape Town, Stinkwater 1246, in monte Diaboli 977 (Bolus 2996).

Phylica rigida Eckl. et Zey. 1024!; Harv. et Sond., Flor. Cap. I., pag. 490.

Cederberge: (leg. Dr. Shaw).

Phylica trachyphylla Sond. in Harv. et Sond, Flor. Cap. I., pag. 492.

Eckl. et Zey. 1001! sub Truchocephalo.

Outeniqua Mnts.: Montagu Pass 282.

In clivis saxosis montis Winterhoeksberg Tulbaghensis (leg. H. Bolus 5147). 
Phylica bicolor Linné., Mant., 208 sec. Harr. et Sond., Flor.

Cap. I., pag. 498.

Herb. Norm. Austr.- Afr. 187!

In ericetis in monte Ninzenberg (leg. H. Bolus 4536).

Phylica ericoides Linn., Spec. plant., pag. 28 sec. Harv. et Sond., Flor. Cap. I, pag. 499.

Bot. Mag. 224!

Eckl. et Zey. 1014!

Promontorium Bonae Spei: Campsbay 1600.

Phylica parvifiora Linn., Mant., 209 sec. Harv. et Sond., Flor. Cap. I., pag. 499.

Eckl. et Zey. 1020!

Promontorium Bonae Spei: in dunis arenosis „Cape Hats" dictis (leg. H. Bolus 3267).

Phylica eriophoros Berg. Pl. Cap. p. 52 sec. Harv. et Sond., Flor. Cap. I., pag. 500.

Promontorium Bonae Spei: Cape Town, Cape flats 2020; Nuizenberg (leg. Bolus 4524).

var. Bergiana Sond. in Harv. et Sond., Flor. Cap. I., pag. 500. Eckl. et Zey. 1036! sub Phylica rosmarinifolia.

WORCESTER: Brandvley 2386, 2387.

Phylica cephalantha Sond. in Harv. et Sond., Flor. Cap. I, pag. 501.

In arenosis prope Groenekloof (leg. Bolus 4262); in montosis circa flum. Hexriver (leg. Bolus 5146), in dunis capensibus prope urbem Cape Town (leg. Bolus nr. ?)

Phylica gnidioides Eckl. et Zey. 1037!; Harv. et Sond., Flor. Cap. I, pag. 501. 
In rupestribus prope Grahamstown (leg. Mac Owan 778).

Noltea africana Reichenb., Consp. n. 3800 sec. Harv. et Sond., Flor. Cap. I, pag. 478.

Herb. Norm. 89! Drege! sub Willemetia africana Brogn.; Eckl. et Zey. 995!

WORCESTER DISTR.: Hexrivervaley 2814.

Helinus ovatus E. Mey. in Drege!; Harv. et Sond., Flor. Cap. I, pag. 479; Oliv., Flor. of Trop. Afr., I, pag. 384.

Herb. Norm. 149! Eckl. et Zey. 996! sub Willemetia scandente.

NataL: Arndolsfarm New Castle 7036; Inanda (leg. J. Wood).

Transvaal: Pretoria Kuduspoort 4647; Makapansberge 5502; Houtbosh 6395.

var. rotundifolius Sond., in Harv. et Sond., Flor. Cap., I, pag. 479.

NATAL: Maritzburg 7505.

\section{AMPELIDEAE}

Vitis fragilis $\mathbf{E}$. Mey, sub Cisso in herb. Drege!; Harv. et Sond., Flor. Cap. I, pag. 249.

Natal: Durban 8762 ; Umbilo ad catarrhactam 8152.

Vitis capensis Thunb., Flor. Cap., pag. 212., Harv. et Sond .

Flor. Cap. I, pag. 249.

Eckl. et Zey. 427! Drege!

Promontorium Bonae Spei: Rondebosh 1653. 
NATAL: Intschanga 7912; Durban 9021.

Outeniqua mnts.: Montagu Pass 274.

var. Dregeana Kunth. in Harv. et Sond., Flor. Cap. I, pag. 250. Drege 7526 ! Krauss!

Natal: Camperdown 7771; Durban 9022.

Outeniqua Mnts.: Montagu Pass 270, 271.

Vitis Thunbergii Eckl. et Zey. 430! sub Cisso, Harv. et Sond., Flor. Cap. I, pag. 250.

Drege 7524 a! 7425 a!

Outeniqua mants.: Montagu Pass 273.

Natal: Umgeni 9020; Durban 9018, 9019; Drakensberg: Van Reenenspass 7218.

Transvaal: Houtbosh 5565.

Vitis paucifora Burch. Cat. 3009 sub Cisso sec. Harv. et Sond., Flor. Cap. I, pag. 251.

Graaff Reinet: (leg. H. Bolus 291).

Vitis cuneifolia Eckl. et Zey. 431! sub Cisso; Harv. et Sond., Flor. Cap. I, pag. 251.

Cissus inaequilaterus E. Mey. in Drege!

NatAL: 6978; Inanda 8385, Camperdown 7691, 7692, Drakensberg Mountainprospect 6979, 6980; Inanda (leg. Wood).

Transvaal: Boshreld inter Elandsriver et Klippan nr.?; Pretoria Derde Poort 4784.

Vitis erythrodes Fresen. in Mus. Senek., II, pag. 284 sec. Oliver, Flor. of Trop. Afr. I, pag. 401.

Transvaal: Hogge Veld inter Porter et Trigardsfontein 6597 .

var. ferruginea Baker in Oliver, Flor. of Trop. Afr. I, pag. 402. 
TransvaAl: Makapansberge Strejdpoort 5495; Pretoria Aapies Poort 4233, Wonderboompoort 4577; Houtbosh 5562 .

Vitis rhomboidea E. Mey. in Drege sub Cisso sec. Harv. et Sond., Flor. Cap. I, pag. 252.

Natal: Maritzburg 7503; Durban 9()16, 9017.

var. transvaalensis nov. var.

caule, petiolis, petiolulis foliisque subtus ad nervos prominentes dense fulvo-villosis.

Transvaal: Houtbosh 5564.

Vitis cirrhosa Thunb., Flor. Cap., pag. 212; Cissus cirrhosus Pers., Ench. I, pag. 142; Harv. et sond., Flor. Cap. I, pag. 252.

Eckl. et Zey. 429 !

NataL: Umbilo ad catarrhactam 8149; Drakensberg Biggarsberge 7099.

TransvaAl: Houtbosh 6396.

var. transvaalensis nov. var.

foliolis obovatis, $10-20 \mathrm{~mm}$. lg. petiolulatis.

Transvaal: Pretoria Wonderboompoort 4578.

Vitis hypoleuca Harv. sub Cisso in Harv. et Sond., Flor. Cap. I, pag. 252.

Transvaal: Houtbosh 6398.

\section{Vitis natalitia nov. sp.}

V. ramis, petiolis pedunculisque angulatis, striatis, dense setoso glandulosis; foliis $30-50 \mathrm{~mm}$. lge. petiolatis, pedatifidis, pinnis externis pedatis pinnatisve, pinna media exteriores multo superante pinnata; foliolis omnibus aequalibus, petiolatis, lami- 
nibus ovato-lanceolatis, angustatis, inaequilateris, mucronato-serratis, utrinque glabris, subcoriaceis, 25-35 mm. lgis., $20-24 \mathrm{~mm}$. Itis.; stipulis lanceolatis, margine laxe setoso-glandulosis, 10-15 lgis., 5-7 mm. Itis.; flores in cymas multoramosas 10-12 cm. longas dispositi, pedunculo primario 11-16 cm. lgo, secundariis $3-4 \mathrm{~cm}$. lgis.; bacca ovoidea, trisperma, glabra, $17 \mathrm{~mm}$. lga, $8 \mathrm{~mm}$. Ita.

Viti adenocauli Steud. proxima differt forma structuraque foliorum.

NataL: Ladysmith 7136; Drakensberg Biggarsberge 7098, Vildshill Pinetown 7991.

\section{SAPINDACEAE.}

Cardiospermum Halicacabum Linn., Spec. plant., pag. 925 sec. Harv. et Sond., Flor. Cap., I, pag. 237.

Transvaal: Boshveld Streydpoort 5032.

NATAL: Oakfort Uwhlotiriver 8525; Inanda (leg. J. Wood).

? Cardiospermum canescens Wall., Plant. Asiat. Rar., I, 14, tab. 14 sec. Oliver, Flor. of Trop. Afr. I, pag. 418. Damaraland : (leg. . . . .)

Schmidelia africana DC., Prodrm., I, pag. 610 , S. melanocarpa Arn. et $S$. leucocarpa Arn. in Hook., Journ., III, pag. 153; Harv. et Sond., Flor. Cap., I, pag. 238.

NatAL: Inanda (leg. J. Wood.).

Schmidelia natalensis Sond. in Harv. et Sond., Flor. Cap. I, pag. 239. 
NATAL: Durban 9037, 9039, 9040, 9041, 9042.

Schmidelia Rehmanniana nov. sp.

S. arborescens, ramulis teretibus, glabris, albidis; foliis 4-6 cm. lge. petiolatis; foliolis 3, coriaceis, subtus palidioribus, utrinque glabris, oblongo-cuneatis, basi attenuatis inaequilaterisque, margine leviter revoluto inciso-repandis, nervis primariis et secundariis utrinque prominentibus, in axillis foveolis pilis densis obsitis notatis, foliolo medio $10-15 \mathrm{~mm}$. lge. petiolatato, $6-8 \mathrm{~cm}$. lgo., 4-5 cm. Ito., foliolis lateralibus $6-10 \mathrm{~mm}$. lge. petiolulatis, $5-7 \mathrm{~cm}$. lgis., $3-3.5 \mathrm{~cm}$. Itis.; racemi axillares, compositi, 6-8 cm. lgi., glabri; flores pólygami, glomerati, pedicellis sepala paulo superantibus; sepalis rotundatis, glabris, margine pilosis; petalis multo brevioribus, villoso-barbatis; ovarium albo-villosum, stylus glaber.

Schmideliae rependae Baker in Oliv., Flor. of Trop. Afr., I, pag. 422 proxima, foliolis petiolutatis, praesentia petalorum et a. r. bene distinguenda.

NATAL: Durban 9038.

? Schmidelia magica Baker in Oliver Flor. of Trop. Afr. I, pag. 423.

Transvaal: Houtbosh 5566.

Schmidelia monophylla Presl., Bot. Bem., 40 sec. Oliver, Flor. of Trop. Afr., I, pag. 424 ; Schmidelia Dregeana Sonder in Hàrv. et Sond., Flor. Cap. I, pag. 329.

var. natalitia nov. var.

ramis juvenilibus, petiolis, pedunculis, pedicellis sepalisque pubescentibus, racemis foliis multo longioribus, cum pedunculo $14-20 \mathrm{~cm}$. longis. 
Natal: Inanda (leg. J. Wood.).

Hippobromus alatus Eckl. et Zey. sec. Harv. et Sond., Flor. Cap. I, pag. 241.

Natal: Drakensberg Van Reenenspass 7279; Oakfort Umhlotiriver 8430; Camperdown 7767, 7690; Arndolfsfarm New Castle 7029; Umgeni 9043; Inanda (leg. J. Wood.).

Kaffraria distr.: (leg. T. Cooper w. 71).

Pappea capensis Eckl. et Zey., En. Pl. Afr. Austr., pag. 53; Sapindus Pappea Sond. in Harv. et Sond., Flor. Cap. I, pag. 241.

Hook., Icon. plant., tab. 352 !

Kaffraria Distr.: (leg. T. Cooper nr.?)

Dodonaea Thunbergiana Eckl. et Zey., 419 sec. Harv. et Sond., Flor. Cap. I, pag. 242.

IN MONTIBUS SUPRA WORCESTER 2524, 2525.

Ceres Distr.: Michelspass 2336.

WORCESTER: Brandvley 2383.

BAKKEFELD: 3091, 3095.

Pteroxylon utile Eckl. et Zeh. sec. Harv. et Sond., Flor. Cap. I, pag. 243.

Harv., Thes. Cap. tab. 17 !

forma: robusta nov. form.

folia $22-30 \mathrm{~cm}$. longe petiolata, foliola $7 \mathrm{~cm}$. lga., $2.5 \mathrm{~cm}$. lata.

Transvaal: Houtbosh 6502.

Aitonia capensis Linn. f. Suppl., 303 sec. Harv. et Sond., Flor. Cap. I, pag. 243.

Somerset: (leg. Mac Owan n. 1412). 
Melianthus major Linn., Spec. plant., pag. 892 sec. Harv. et Sond., Flor. Cap., I, pag. 367.

Bot. Reg., tab. 45 !

Promonturium Bonae Spei: Cape Town, 1552.

Melianthus comosus Vahl., Symb., III, pag. 86 sec. Harv. et Sond., Flor. Cap., I, pag. 367.

Bot. Mag., tab. 301!

KaRROo: Grootfontein 3003, 3050; Witteberge Maggisfontein 2951.

Somerset East.: ad ripes fluminis Klyn Vischriver (leg. Mac Owan).

? Melianthus Dregeana Sond. in Harv. et Sond., Flor. Cap. I, pag. 368.

Transvaal: Hogge Veld Sandspruit 6869.

Greyia Sutherlandi Hook. et Harv. in Proceed. of the Dublin University Zoolog. and Botan. Assoc., I, part 2, pag. 138, tab. 13-14; Harv. et Sond., Flor, Cap. II, pag. 308, 309. Harv., Thes. Cap. I, tab. 1 !

NataL: Drakensberg Van Reenenspass 7200, Biggarsberge 7077, Inanda, culta in horto cl. dom. J. Wood.

Greyia Radlkoferi nov. sp.

G. frutescens (arborescens?), ramulis teretibus, albo-tomentosis; folia alterna, 4-5 cm. lge. petiolata, petiolis albo-tomentosis, lata basi subvaginantibus, laminis subrotundatis, basi cordatis, multilobulatis, inaequaliter crenato-serratis, supra stellato-adpresse-pilosis, subtus dense albotomentosis, $5-7$ pedatinerviis; flores rosei (lutei?) 
sessiles in racemos densos, axillares, $2-4 \mathrm{~cm}$. (?) longos, congesti; pedunculis glabris, bracteis lanceolatis; calyx quinque-partitus, brevis, persistens, laciniis acutis; petala 5, spathulata, hasi angustata, 13-15 mm. lga.; discus cupularis, quinquelobus, lobis bidentatis, apice staminodiis glanduliferis coronatis; stamina 10, intra discum inserta, filamentis filiformibus, $15-20 \mathrm{~mm}$. lgis.; antherae ovatae, didymae; ovarium oblongum, profunde 5 -sulcum, in stylum gracilem $10 \mathrm{~mm}$. lgum. attenuatum; capsula membranacea, septicide in follicula 5 partibilis, $10-12 \mathrm{~mm}$. Iga. (immatura?)

TransvaAL: Houtbosh 6404.

\section{Bersama lucens Szysz.}

Natalia lucens Hochst. in Flora, 1841, pag. 663; Harv. et Sond., Flor. Cap. I, pag. 369. Rhaganus lucidus E. Mey. in herb. Drege!

Natal: Durban 9057 ; inter Pintown et Umbilo 8042.

\section{ANACARDIACEAE.}

Rhus rosmarinifolia Vahl. Symb., III, pag. 50 sec. Engler, Anac. in DC. Mon. Pban., IV, pag. 404; Harv. et Sond., Flor. Cap., I, pag. 506.

Drege 231! 2227! 6812 !

Promontorium Bonae Spei: Cape Town, Stinkwater $1346,1536$.

Rhus stenophylla Eckl. et Zey. 1094 sec. Engler, Anac. in DC., Mon. Phan., IV, pag. 404.

Drege 6813!

Promontorium Bonae Spei: Cape Town 1533. 
Rhus angustifolia Linn., Spec. Plant., 882 sec. Engler, Anac. in DC., Mon. Phan., VI, pag. 405.

Eckl. et Zey., 1092!, Drege 6810 A !

Promontorium Bonae Spei: Cape Town 1532, 1534; in monte Diaboli 1142.

WorCester: Brandvaley 2382; Hexrivervaley 2817.

HeXriverberge: Axellsfarm 2719.

Rhus obovata Sond., in Harv. et Sond., Flor. Cap. I, pag. 508, Engler Anac. in DC., Mon. Phan., IV, pag. 406. Kaffraria : (leg. J. Cooper 407).

Rhus tomentosa Linn., Spec. plant., 382 sec. Engler, Anac. in DC., Mon. Phan., IV, pag. 407; Harv. et Sond., Flor. Cap., I, pag. 508.

Eckl. et Zey. 1109!

Promontorium Bonae Spei: Cape Town 1530, 1531;

Stinkwater 1348, 1349.

WORCESTER: in montibus 2519.

Somerset East.: in montibus Zwagers Hock. (leg. Mac Owan 296).

Rhus incisa Linn. fil., Suppl., 183 sec. Engler, Anacard. in DC., Mon. Phan., IV, pag. 408; Harv. et Sond., Flor. Cap., I, pag. 509.

Eckl. et Zey., 1112!, Drege 6793B!

WORCESTER: in montibus 2518.

Rhus dissecta Thunb., Flor. Cap., 267 sec. Engler, Anac. in DC., Mon. Phan., IV, pag. 408; Harv. et Sond., Flor. Cap. I, pag. 509.

Eckl. et Zey., 1127! sub $R$. argentea.

WORCESTER DISTR. : Hexrivervaley 2821. 
Rhus undulata Jacq., hort. Schoenbr., tab. 346 sec. Engler Anac. in DC., Mon. Phan., IV, pag. 410; Harv. et Sond., Flor. Cap. I, pag. 518.

Eckl. et Zey., 1117! sub $R$. aglaophylla, 1125! sub $R$. excisa, 1124 ! sub. $R$. micrantha.

WORCESTER DISTR.: in montibus 2517.

KARROO: Witteberge, Maggisfontein 2912.

Rhus excisa Thunb., Flor. cap., 264 sec. Engler Anac. in DC., Mon. Phan., IV, pag. 410 ; Harv. et Sond., Flor. Cap. I, pag. 518.

NataL: Drakensberg Tugelariver Colenso 7178 (det. Engler).

Somerset East.: in sylvis montis Boschberg (1. Mac Owan 514).

Rhus glauca Desf. Arb., I, 326 sec. Engler Anac. in DC., Mon. Phan., IV, pag. 411; Harv. et Sond., Flor. Cap., I. pag. 516.

Eckl. et Zey. $1121 ; 1122$ sub R. Thunbergiana. Promontorium Bonae Seer: Cape Town 1528, 1529.

Rhus outeniquensis nov. sp.

Ramulis novellis glabris; foliis $10-12 \mathrm{~mm}$. lge. petiolatis, petiolis vix alatis; foliolis brevissime petiolulatis, obovato-oblongis, apice obtuso-acuminatis subemarginatisque, inferne cuneatim angustatis, margine integerrimo leviter revolutis, coriaceis, nervis lateralibus subtus levissime prominulis, medio $6-7 \mathrm{~cm}$. lgo., $20-25 \mathrm{~mm}$. lto., lateralibus $4-4.5 \mathrm{~cm}$. lgis., $15-18 \mathrm{~mm}$. Itis.; paniculis axillaribus, minute puberulis $3-3.5 \mathrm{~cm}$. lgis.; bracteolis $1 \mathrm{~mm}$. lgis. pedicello dimidio brevioribus, puberulis; calycis segmentis ovatis mi- 
nute puberulis; petalis oblongis, $1-1.5$ mm. lgis., sepala duplo vel triplo superantibus; drupa subglobosa glabra.

Rh. scytophyllae proxima differt forma foliolorum petiolulatorum paniculisque foliis $2-3$ longioribus, etc.

Outeniqua Mnts.: Montagu Pass 272.

Rhus ciliata Licht, in herb. Willd. sec, Engler Anac. in DC., Mon. Phan., IV, pag. 418.

Drege 6804 A!

Hopetown : 3223.

Griqualand W.: Kimberley 3433.

Oranje Fr. S'T.: Olifantsfontein 3518.

Rhus coriacea Engler Anac. in DC., Mnn. Phan., IV, pag. 418, Transvaal: Boshveld Klippan 5329 (det. Engler); Pretoria 4745 (det. Engler).

Rhus natalensis Bernh. in Krauss. Beitr., 46 sec. Engler Anac., in DC., Mon. Phan., IV, pag. 421; Harv. et Sond., Flor. Cap. I. pag. 515.

NataL: Durban 9034 (det. Engler), 8956, (Wood 378).

Rhus crenata Thunb., Flor. Cap., 266 sec. Engler Anac. in DC., Mon. Phan., IV, pag. 422; Harv. et Sond., Flor. Cap. I, pag. 512.

Eckl. et Zey., 1123!

Natal: inter Pintown et Umbilo 8072 (det. Engler). Oakfort Umblotiriver 8429.

Rhus Rehmanniana Engler. Anac. in DC., Mon. Phan., IV, pag. 422 .

Transvaal: Houtbosh 5560 (det. Engler); 5561 (det. Engler), 5568 (det. Engler). 
Natal: Arndolfsfarm New Castle 7051 (det. Engler) Drakensberg Biggarsberge 7053 (det. Engler).

Rhus acutidens Engler. Anac. in DC., Mon. Phan., IV, pag. 423. Transvaal: Houtbosh 5558 (det. Engler).

Rhus villosa Linn. f. Suppl., 183 sec. Engler Anac. in DC., Mon. Phan., IV, pag. 424; Harv. et Sond., Flor. Cap. I. pag. 510.

Eckl. et Zey., 1098!

BAKKEFELD : 3071.

Outeniqua Mnts.: Montagu Pass 181, 184.

Oranje Fr. St.: Mudriverdrift 3593.

Transvaal: Pretoria 4741 (det. Engler); Makapans-

berge Streydpoort 5555 (det. Engler); Boshveld Klippan 5326 (det. Engler); Houtbosh 5574 (det. Engler), 5575 (det. Engler).

Somerset East: in dumetis (leg. Mac Owan 505).

var. gracilis Engler l. c. pag. 425.

Transvaal: Pretoria 4742 (det. Engler).

NATAL: Drakensberg Laingsnek 6960, Mountainprospect 7009; Maritzburg 7489; Umgeni 7439.

var. glabrata Sond. in Harv. et Sond., Flor. Cap., I. pag. 510.

Promontorium Bonae Sper: Cape Town, Stinkwater 1350; Cape flats 2205.

Rhus tridentata Sond. in Harv. et Sond., Flor. Cap., I, pag. 425; Engler Anac. in DC., Mon. Phan., IV, pag. 425 .

NataL: Inanda 8178 (det. Engler). 
Rhus refracta Eckl; et Zey., nr. 1103!; Engler, Anac. in DC., Mon. Phan., IV, pag. 427; Harv. et Sond., Flor. Cap. I, pag. 511.

Natal: Arndolfsfarm New Castle 7052 (det. Engler) Drakensberg, Biggarsberge 7054.

Rhus incana Engler Anac. in DC., Mon. Phan., IV, pag. 428. Transvaal: Boshveld Klippan 5325 (det. Engler); Elaudsriver Neu Halle 4913 (det. Engler).

Rhus divaricata Eckl. et Zey., nr. 1106 sec. Engler, Anac. in DC., Mon. Pban., IV, pag. 429.

var. fulvescens Engler. 1. c.

Transvaal: Hogge Veld Trigardsfontein 6705 (det. Engler).

Rhus pyroides Burch., Trav., I, 340 sec. Engler Anac. in DC., Mon. Phan., IV, pag. 430; Harv. et Sond., Flor. Cap. I, pag. 511.

Promontorium Bonae Spei: Houtbay 1619; Cape Town 1526.

Drakenstein BG.: Klapmuth 2267.

Rhus glaucovirens Engler Anac. in DC., Mon. Phan., IV, pag. 432.

Transvaal: Pretoria 4740 (det. Engler); (5618 leg. H. Bolus sub R. Zeyheri Bolus! non Sond.).

Rhus mucronata Thunb., Flor. Cap., 264 sec. Engler Anac. in DC., Mon. Phan., IV, pag. 432; Harv. et Sond, Flor. Cap. I, pag. 513.

var. Burmanni Sond. in Harv. et Sond., Flor. Cap. l. c. Promontorium Bonae Spei: Cape Town 1527. 
var. Jacquini Sond. 1. c.

Promontorium Bonae Spei: Cape Town, Cape flats 2203.

Rhus Zeyheri Sond. in Harv. et Sond., Flor. Cap. I, pag. 433;

Engler Anac. in DC., Mon. Phan., IV, pag. 433.

var. dentata Engler 1. c.

NATAL: Drakensberg Laingsnek 6942 (det. Engler).

Rhus grandifolia Engler Anac. in DC., Mon. Phan., IV, pag. 434.

NATAL: Inanda 8175 (det. Engler).

Rhus dentata Thunb., Flor. Cap., 266 sec. Engler Anac. in DC., Mon. Phan., IV, pag. 435; Harv. et Sond., Flor. Cap., I. pag. 513 p. p.

Eckl. et Zey., 1126!

NataL: Camperdown '1765, Inanda 8173, 8174 (det.

Engler), Intschanga nr. ? (det. Engler).

Kaffraria: leg. Cooper nr. 275, 422.

Rhus Sonderi Engler Anac. in DC., Mon. Phan., IV, pag. 435.

var. glaberrima Engler 1. c.

Transvaal: Pretoria 4744 (det. Engler).

var. pilosa Engler 1. c.

Transvaal: Pretoria 4743 (dat. Engler).

NATAL: Drakensberg Laingsnek 6945 (det. Engler).

var. pilosissima Engler 1. c.

Transvaal: Hogge Veld Pages hôtel 6863 (det. Engler). 
Rhus erosa Thunb., Flor. Cap., 263 sec. Engler, Anac. in DC., Mon. Phan., IV, pag. 439; Harv. et Sond., Flor. Cap. I, pag. 516.

Eckl. et Zey. 1133!; Burchell Cat. 2697!

Oranje Fr. St.: Witteberge Kadziberg 3981; Bloemfontein $3795,3897$.

Craddock distr.: leg. T. Cooper 487.

var. subintegra nov. var.

foliis margine subintegris.

Oranje Fr. St.: Bloemfontein 3832.

Rhus transvaalensis Engler Anac. in DC., Mon. Phan., IV, pag. 440.

Transvaal: Houtbosh 5559 (det. Engler), 5572 (det. Engler).

Rhus viminalis Vahl. Symb., III, 50 sec. Engler Anac. in DC., Mon. Phan., IV, pag. 442 ; Harv. et Sond., Flor. Cap, I, pag. 515.

Transvaal: Boshveld Elandsriver Neu Halle 4883 (det. Engler); Houtbosh 5570 (det. Engler), 5571 (det. Engler).

var. pendulina Sond. in Harv. et Sond., l. c.; Engler l. c. ROGGEFELD : Korlanskloof 3180.

var. Gerrardi Engler 1. c.

NataL: Drakensberg Coldstream 6883 (det. Engler); Lainsgnek 6943.

Rhus Gueinzii Sond. in Harv. et Sond., Flor. Cap. I, pag. 442; Engler Anac. in DC., Mon. Phan., IV, pag. 442. 
Transvaal: Bosliveld inter Elandsriver et Klippan 5143 (det. Engler); Klippan 5327, 5328 (det. Engler); Makapansberge Streydpoort 5554 (det. Engler).

Rhus laevigata Linn., Spec. plant., 1672 sec. Engler Anac. in DC., Mon. Phan., IV, pag. 443; Harv. et Sond., Flor. Cap., I., pag. 264.

Transvaal: Houtbosh 5569 (det. Engler).

NATAL: Inanda 8177, 8387 (det. Engler); Durban 9030, 9033 (det. Engler).

Outeniqua Mnts.: Montagu Pass 182.

Rhus lancea Linn. fil. Suppl., 184 sec. Engler, Anac. in DC., Mon. Phan., IV, pag. 444; Harv. et Sond., Flor. Cap., I, pag. 514.

Transvaal: Pretoria 4747 (det. Engler); Boshveld inter Elandsriver et Klippan 5141, 5142 (det. Engler). BAKKEFELD: Ceres 3075. Hexrivervaley: Groote Tafelberg 2772.

Oranje Fr. Sт.: Korannaberg 4006, Bloemfontein 3880. KaRRoo: Witteberge, Maggisfontein 2911.

Rhus Dregeana Sond. in Harv. et Sond., Flor. Cap. I., pag. 516; Engler Anac. in DC., Mon. Phan., IV, pag. 445. Rhus lancea E. M. in Drege!

Promontorium Bonae Sper: Cape Town, Stinkwater 1347; in monte Diaboli 1141.

Rhus gracillima Engler Anau. in DC., Mon. Phan., IV, pag. 445. Transvaal: Boshveld Menaarsfarm 4882 (det. Engler).

Rhus villosissima Engler. Anac. in DC., Mon. Phan., IV, pag. 447.

Transvaal: Houtbosh 5557 (det. Engler). 
Rhus discolor E. Mey. in Drege!, Engler Anac. in DC., Mon. Phan., IV: pag. 447; Harv. et Sond., Flor. Cap., I, pag. 507.

Promontorium Bonae Spei: Cape Town, Stinkwater 1351, Cape flats 2202?

Transvaal: Hogge Veld Bronkersspruit 6575 (det. Engler).

var. paucinervia Engler l. c.

Transvaal: Hogge Veld Pages hôtel 6864 (det. Engler).

var. brevifolia Engler 1. c.

NataL: Drakensberg Laingsnek 6941 (det. Engler); Coldstream 6896.

Anaphrenium paniculosum Engler, Anac. in DC., Mon. Phan. IV, pag. 358 ; Rhus paniculosa Sond., in Harv. et Sond., Flor. Cap. I, pag. 522.

Transvaal: Boshveld Menaarsfarm 4881 (det. Engler), inter Elandsriver et Klippan 5154 (det. Engler); Makapansberge Streydpoort 5556 (det. Engler).

Anaphrenium argenteum ฐ. Mey. in Drege sec. Engler Anac. in DC., Mon. Phan., IV, pag. 360, Rhus Thunbergii Hook. Jeon. 595.

HeXriverberge: Axallsfarm 2720.

Ceres Distr.: Michelspass 2342.

Drakenstein BG.: Bainskloof 2328.

Loxostylis alata Spreng, fil. sec. Engler Anac. in DC., Mon. Phan., IV, pag. 352.

Durban: Berea (leg. J. Wood nr. 362).

Smodingium argutum Sond. in Harv. et Sond., Flor. Cap. I., p. 523 ; Engler Anac. in DC., Mon. Phan., IV, p. 355. 
NATAL: Great Noodsberg (leg. J. Wood) (det. Engler).

Odina discolor Sond. in Linn., XXIII, I, pag. 25 sec. Engler, Anac. in DC., Mon. Phan., IV, pag. 272; Harv. et Sond., Flor. Cap. I., pag. 524.

Transvaal: Pretoria 4736, 4737, 4738 (det. Engler).

Odina edulis Sond, in Harv. et Sond., Flor. Cap. I., pag. 503. Engler, Anac., in DC., Mon. Phan., IV, p. 272.

Transvaal: Houthosh 6499 (det. Engler); Pretoria 4735 (det. Engler).

NATAL: Inanda 8179 (det. Engler).

var. glabrescens Engler l. c.

Transvaal: Pretoria 4739 (det. Engler).

Sclerocarya caffra Sonder in Linn., XXIII, pag. 26 sec. Engler, Anac. in DC., Mon. Phan., IV, pag. 257, Harv. et Sond., Flor. Cap. I., pag. 525.

TransvaAL: Makapansberge Streydpoort, 5374 (det. Engler).

var. dentata Engl. 1. c. pag. 258.

Transvaal: Boshveld inter Elandsriver et Klippan 5140 (det. Engler), Pretoria 4734 (det. Engler).

Vindobonae 20 Augusti 1887. 


\section{INDEX}

specierum et synonymorum

Polypetalarum Disciflorarum Rehmannii.

A ridocarpus galphimiaefo lius Juss. . . . . 2

$"$ natalitius Juss. . . . 2 pruriens Juss. . . . 2 Adenandra cuspidata Mey. . 23 v. glabra Sond. . . 23 v. villosa Sond. . . 23 humilis E. et Z. . . 24 v. glabra Sond. . . 24 marginata $R$. et Sch. 24 umbellata Willd. . . 24 v. glandulosa B. et W. 24 uniflora Willd. . 24

Agathosma ambigua Sond. 27

" biophylla E. et $Z$. . 25

" bifida E. et Z. . . . 25

" cerefolium B. et W. 26

" v. glabrata S. 27 chortophila E. et Z. 27 ciliata Link. . . 27 commutata Bolus. . 27 cuspidata B. et W. . 27 v. glabra Sond. 27 hirta B. et W. . . 25 imbricata Willd. . . 25

v. acuminata S. 26

"v. reflexa Sond. 26 marifolia E. et Z. . 26 v. lanceolata S. 26 patentissima $E$. et $Z .27$ $n$ » rugosa Link. . 26
Agathosma v. glabra Szysz. 26 " " v. lancifolia Sz. 26 $" \quad " \quad$ v. pubescens Sz. 26 umbellata Sond. . . 25 Aitonia capensis L. . . . 48 Anaphren. argenteum E. M. 59 " paniculos. Engl. . . 59 Apodytes dimidiata E. M. . 31 Augea capensis Thunb. . . 4

Banisteria Kraus. Hochst. 2 Barosma lanceolata v. nata-

lensis Sond. . . 25 Kraussiana Buch. . 25

$"$ scoparia E. et $Z$. . 25

"graveolens $E$. et Z. 25

" ovata B. et W. . . . 24

$" \quad$ v. vera Szysz. 25

$" \quad$ " v. cuneata Sz. 25

$n$ pulchella B. et W. . 24 Bersama lucens Szysz. . .50

Calodendron capense Th. . 22 Cardiospermum canesc. W. 46 Halicacabum L. . . 46 Cassine barbara L. . $\quad 32$ " capensis L. . . . . 32 " ilicifolia Hochst. . . 31

" Maurocenia L. . . 33

" scandens E. et Z. . . 32 
Cassine scandens v. latifolia

Sond. . . . . 33

Cassinopsis capensis Sond. . 31

Catha edulis Forsk. . . . 32

Forskalii Rich. . . 32

Celastrus acuminatus $L$. 33

"buxifolius L. . . . . 34

, campestris $E$. et $Z . \quad 36$

"huillensis Welw. . . 35

$"$ ilicinus $E$. et $Z$. . . 34

" luteolus Dell. . . . 35

" mucronatus E. et Z. 33

"nemorosus E. et Z. 35

" ovata E. $M$. . . . 31

" polyacanthus Sond. . 33

" pyracanthus $L$. . . 36

" sinuato-dentatus Hoch. 35

" tenuispinus Sond. . . 33

" undatus Th. . . 34

"Zeyheri Sond. . . 33

Clausena anisata Oliv. . . 28

" inaequalis Benth. . 28

Cissus cirrhosa Thunb. . . 44

" cuneifolia $E$. et $Z$. . 44

" fragilis E. M. . . 43

" inaequilatera E. M. 44

" pauciflora Burcl. . 44

" rhomboidea E. M. . 44

" Thunbergii E. et $Z .44$

Coleonema gracile $E$. et $Z$. 23 $"$ pulchrum Hook. . . 23

"virgatum $E$. et $Z$. .23

Commiphora Rehman. Engl. 30

Diosma scabra Lam. .

succulenta Berg.

v. Lamarckjana Sond. 22

" virgata Mey. . . . 23

" vulgaris Schl. . . . 22

v. longifolia Sond. . 23

Diporidium atropurpureum

Wendl. . . . . 29

natalitium Meissn. . 29

arboreum Wendl. . 29
Dodonaea Thunbergiana E. et Z. . . . . 48

Elaeodendron capense $\mathrm{E}$. et Z. . . . . . 37

„ confertifolium Tul. $\quad 37$

$"$ v. leptocarpum Sond. 37

$"$ glaucum Szysz. . . 36

" Rehmannii Szysz. . . 37

Ekebergia Meyeri Presl. . . 30

" capensis DC. . . . . 31

Empleurum serrulatum Ait. . 27

Erodium moschatum Willd. 8 var. ciliatum Harv. . 8

Eumorpha nobilis $E$. et Z. 13 tenuiloba E. et $Z$. 13 Erythroxylon cafrum Sond. 2

$"$ emarginatum S. et T. 2

$"$ pictum E. M. . . . 2

Fagara capensis Thbg. . . 27

Geranium canescens L'H. . 7 ciliatum Cav. . . . 8

" incanum L. . . . . 7

" incanum E. MI. . . 7

" ornithopodum E. et Z. 7

" sericeum Harv. . . . 7

Greyia Radlkoferi Szysz. . 49

"Sutherlandi Hook. et Harv. .

Gymnosporia acuminata L. 33 buxifolia (L.). . . . 34

$" \quad$ "v.genuina Sond. 34

$" \quad$ v. laxiflora S. . 34

$" \quad$ " v. venenata S. . 34

" huillensis (Welw.). . 35

" laurina (E. et Z.). . 35

$" \quad$ luteola (Del.). . . . 35

$"$ nemorosa (E. et Z.). 35

" polyacantha (Sond.). 33

" Rehmannii Szysz. . . 34

$"$ tenuispina (Sond.). . 33

» undata (Thunb.). . . 34 
Gymnosporia Woodii Szysz. 35 Zeyheri (Sond.). . . 33 Hartogia capensis Thunb. . 32 $" \quad$ v. lanceolata S. 32 v. multiflora E. et $\mathrm{Z}$. 32 lanceolata $E$. et $\dot{Z}$. 32 Helinus ovatus E. M. . 43 Hippobromus alatus E. et Z. 48 Hoarea pinnata Eckl. et Zey.

Ilex capersis sond. . . . 31 Impatiens capensis Th. . . 21 Isopetalum dentatum E. et Z. 13

Lauridia reticulata E. et Z. 33 Linum thesioides Bart. . . 1

Thunbergii E. et Z. . 1 Loxustylis alata Spreng. . 59

Macrostylis lanceol. E. et Z. 22 "villosa Sond. . . . . 22 Maurocenia capensis Sond. 33 Melia Azederach L. . . . 30 Melianthus comosus Vahl. . 49 "Dregeanus Sond. . 49 $"$ major L. . . . . . 49 Methyscophyllum glaucum $\mathrm{E}$. et Z. . . . . . 32 Monsonia angustifolia $E$. M. 6 $"$ attenuata Harv. . . 7 " biflora $E$. et $Z$. . . 6

" Burkeana Planch. . . 6

" ovata Cav. . . . 6 v. biflora Harv. . . . 6 v. lancifolia Szysz. . 6 "praemorsa $E . M$. . 6 " speciosa L. . . . . 47 Iyuris inaequalis Presl. . 8 Myrrhidium lacerum $E$. et $Z$. . . . . . 11 longicaule $E$. et $Z$. . 12 " triangulare $E$. et $Z$. . 12
Tag.

tucens Hochst.

Noltea africana Reich. . . 43

Ochna arborea Burch. . . 29

$"$ atropurpurea DC. . . 29

" $\quad$ v. natalitia H. 29

Rehmannii Szysz. . . 28

Udina discolor Sond. . . . 60

" edulis Sond. . . . 60

$" \quad$ v. glabres. Eng. 60

Oxalis bifida Th. . . . 19

, bifurca Lodd. . . . 19

v. incana Harv. . . . 19

bisulca E. M. . . . 20

calcaria E. et Z. . 18

caprina L. . . . . 21

ceratilis E. M. . . 21

cernua Th. . . . 21

corniculata L. . . 21

commutata Sond. . . 17

cuprea Sond. . . . 19

dentata $E$. et $Z$. . . 21

erubescens E. M. . 17

flava L. . . . . 20

v. Thunbergiana L. 20

glabra Th. . . . 16

v. pusilla Sond. . . 16

hirta L. . . . . 20

hirtella Jacq. . . . 20

imbricata E. et Z. . 18

v. rosea Sond. . . 18

lanata L. . . . . 19

livida Jacq. . . . . 21

livida Eckl. . . . 19

lutea $v$. marginata S. 18

luteola Jacq. . . . 18

macrophylla Horn. . 21

Mariae Szysz. . . . 18

multiflora Jacq. . 20

obtusa Jacq: . . . . 19

" polyphylla Jacq. . . 16

v. filifolia Sond. . . 16

" pulchella Jacq. . . . 17

v. glabrata Sond. . . 17 
Oxalis punctata L. . . . . i

" purpurea Th. . . . 17

" pusilla Jacq. . . . . 16

" revoluta E. M. . . 16

" rigidula $E$. et $Z$. . . 18

" mbella Jacq. . . . 20

" semiloba Sond. . . 21

" sericea L. . . . 20

"spec.v.purpurea E.M. 17

" strumosa E. M. . . 18

"Smithii Sond. . . 19

v. latifolia Sond. . . 20

tenuifolia Jacq. . . 17

tubiflora Jacq. . . . 20

variabilis Lindl. . . 17

v. nana Sond. . . 17

$"$ versicolor L. . . . 16

$"$ tenella E. et $Z$. . . 17

" tomentosa L. . . . 20

Pappea capensis E. et Z. . 48 Pelargonium abrotanifolium Jacq. . . . . . 11

$"$ acetosum Ait. . 14

" aconitifolium E. et Z. 10

" alchemilloides Willd. 13

v. aphanoides Harv. 13

v. dentatum Harv. 13

angulosum Ait. . . 14

v. angulosum Harv. 14

v. truncatum Szysz. . 14

astragalifolium Pers. . 9

v. foliosum Harv. . . 9

v. minus Harv. . . . 9

barbatum Jacq. . . 9

betulinum Ait. . . 14

capitatum Ait. . . . 13

ceratophyllum L'H. . 11

columbinum E. MI. . 12

crispum Ait. . . . 15

cucullatum Ait. . . 14

dissectum E. et Z. . 11

filipendulifol. E. M. 10

flabellifolium Harv. . 10
Pelaron.

P'H. 12

" glaucum L'H. . . . 14

" grossularioides Ait. . 12

v. anceps Harv. . . 12

grassularioides Cooper 13

hispidum Willd. . 15

lanceolatum Cav. . 14

lobatum Willd. . . 9

multicaule Jacq. . , 12

multicaule Cooper. . 12

myrrhifolium Ait. 11

v. fruticosum Harv. . 11

v. lacerum Harv. . . 11

v. longicaule Harv. . 11

ovale Burm. . . . . 12

patulum Jacq. . . 13

v. tenuilobum Harv. . 13

patulum E. M. . . 13

" peltatum Ait. . . 12

v. clypeatum Harv. 13

pinnatum Andr. . 9

polymorphum E. M. 10

pulverulentum Colv. . 9

v. pedicellatum Harv. 10

Radula Ait. . . . 15

ramosissimum Willd. 11

rapaceum Jacq. . . . 9

Rehmannii Szysz. . 8

roseum $E$. et $Z$. . 16

saniculaefolium Willd. 13

scabrum Ait. . . . 15

tabulare Cav. . . 13

triste Ait. . . . 10

v. daucifolium Harv. 10

v. filipendulifolium H. 10

triste E. MI. . . . 10

Tysonii Szysz. . . 8

vitifolium Ait. . . 15

Zeỷheri Harv. . . 10

zonale Willd. . . 14

Phylica bicolor I. . . . . 42

" capitata Thunb. . . 41

$"$ cephalantha Sond. . 42

buxifolia L. . . . 39 
Phylica cylindrica E. et Z. ${ }^{\text {pag. }} 1$ ericoides L. . . . 42 eriophoros Berg. . . 42

$" \quad$ v. Bergiana S. 42 excelsa Wendl.

" $\quad$ v. brevifolia S. 41

"v. laxa Sond. . 41

"v. papillosa S. 41

" v. stricta Sond. 41 fulva $E$. et $Z$. . . 41 gnidioides E. et Z. . 42 lutescens Sond. . . 40 oleoides DC. . . . 40 paniculata Willd. . . 39 paniculata $v$. scabra

Bolus. . . . . . 40 parviflora L. . . . . 42 plumosa Thunb. . . 40 rigida $\mathrm{E}$. et $\mathrm{Z}$. . . 41 rigidifolia Sond. . . 40 rosmarinifolia $E$. et $Z .42$ stipularis L. . . . 39 trachyphylla Sond. . 41 villosa 'Th. . . . . 40

"v. glabrata S. 40 Polyactium daucifolium $E$. et Z. . . . . 10 filipendulifol. E. et Z. 10 sphondylifol. E. et Z. 9

" triste E. et Z. . . . 10

Pterocelastrus obtusus Hoch. 31 macrostylus Hochst. . 31 Pteroxylon utile E. et Z. . 48 f. robusta Sz. . 48 Putterlickia pyracantlı (L.). 36 $"$ verrucosa Sond. . . .36

Rhaganus lucidus E. Mey. 50 Rhamnus prinoides L'Her. . 39 Rhus acutidens Engl. . . 54 aglaophylla $E$. et Z. 52 angustifolia $\mathrm{L}$. . . . 51 argentea $E$. et $Z$. . 51
Rhus ciliata Licht. . . 53 coriacea Engl. . . . 53 crenata Thunb. . . .53 dentata Thunb. . . 56 discolor E. M. . . 59 " v. brevifolia E. 59

" v. paucinerv. E. 59 dissecta Thunb. . . 51 divaricata E. et Z. . 55 v. fulvescens E. 55 Dregeana Sond. . . 58 erosa Thunb. . . . .57 v. subintegra Sz. 57 excisa Thunb. . . . 52 excisa E. et Z. . . .52 glauca Desf. . . . . 52 glaucovirens Engl. . 55 graciliima Engl. . . 58 grandifolia Engl. . . 56 Gueinzii Sond. . . 57 incana Engl. . . . 55 incisa L. . . . 51 laevigata L. . . . 58 lancea L. . . . . 58 lancea E. M. . $\quad 58$ micrantha $E$. et $Z$. . 52 mucronata Thunb. .55 v. Burmannii S. 55 " v. Jacquini S. . 56 natalensis Bernh. . . 53 obovata Sond. . . 51 outeniquensis Szysz. . 52 paniculosa Sond. . .59 pyroides Burch. . . . 55 refracta E. et Z. . 55 Rehmanniana Engl. . 53 rosmarinifolia Vahl. . 50 Sonderi Engl. . . . 56

v.glaberrima E. 56

$"$ v. pilosa Engl. 56

"v. pilosissima Engl. . . . . . . 56 stenophylla E. et Z. . 50 Thunbergii Hook. . 59 
Rhus tomentosa L. . . $\quad .51$ transvaalensis Engl. . 57 tridentata Sond. . . 54 undulata Jacq. . . . 52 villosa L. . . . . . 54 v. glabra Sond. 54 $" \quad$ v. gracilis Engl. 54 vilosissima Engl. . . 58 viminalis Vahl. . . 57 v. Gerrardi E. . 57 $"$ v. pendulina S. 57 Zeyheri Sond. . . . 56 v. dentata Engl. 56 Zeyheri Bolus . . . 55

Salacia Kraussii Hochst. . 38 Sapindus Pappea S. . . . 48 Sclerocarya caffra Sond. . . 60 " $"$ v. dentata . . 60 Schmidelia africana DC. $\quad .46$ " leucocarpa Arn. . 46 " magica Bak. . . . 47

" melanocarpa Arn. . . 46

" monophylla Presl. . . 47

" " v. natalitia Sz. 47

$"$ natalensis Sond. . . 46

^Rehmanniana Szysz. . 47 Scutia Commersoni Brogn. . 39 " indica Brogn. . . 39 Scytophyllum laurinum $E$. et $Z$. . . . . . 35

Smodingium argutum Sond. 59 Soulangia buxifolia $E$. et $Z$. 39 "lutescens $E$. et $Z$. . 40 " paniculata $E$. et Z. . 40 " pinea E. et Z. . . . 40 Sphedamnocarp. galphimiaefolius (Juss.). " $"$ pruriens (Juss.) 2 $"$ form. major Szysz. . 2 "Rehmannii Szysz. . 3

Toddalia lanceolata (Juss.). 28 Triaspis Rehmannii Szysz. . 3
Tribulus terrestris Tribulus terrestris L. . . . 4 v. desertorum Sond. . 4 v. hispidissimus Sond. 4 Trichilia Ekebergia E. M. 30 Trichocephalus stipularis $E$. et $Z$. . . . . . 39 " trachycephalus E. et Z. 41 Turraea obtusifolia Hochst. 30

Vepris lanceolata Juss. . . 28 Vitis capensis Thunb. . . 43

$" \quad$ v. Dreg. Kunth. 44 " cirrhosa Th..... 45

" $\quad$ v.transvaalensis Szysz. . . . . . 45 cuneifolia E. et Z. . 44 erythrodes Fres. . 44

" $" \quad$ v. ferruginea B. 44 fragilis (E. M.) . . . 43

" hypoleuca Harv. . . 45

$"$ natalitia Szysz. . . 45

" pauciflora Burch. . . 44

" rhomboidea E. M. . . 45

$" \quad$ v. transvaalensis Szysz. . . . . 45

"Thunbergii E. et Z. . 44

Willemetia africana Brogn. 43 "scandens $E$. et $Z$. . 43

Ximenia caffra Sond. . . . 31

Zizyphus helvola Sond. . . 38 mucronata Willd. . 38 " " v. glabrata S. 38 " $\quad$ v. pubescens S. 38 " Spina.Christi Willd. 38 Zanthoxylum capense Harv. 27 Thunbergii DC. . . 27 Zygophyllum Commelini $E$. et $Z$. . . . . . 5 
Zygoph. divaricatum E. et ${ }^{\text {pag. }}$ Z. . . . . . 5

"flexuosum E. et Z. . 5 v. cuneatum Szysz. . 5 " glaucum E. M. . . . 5
Zygoph. limosum E. et Z. 5 microcarpum Licht. . 5 microphyllum L. . . 5 Morgsana L. . . . 5 sessilifolium L. . . . 4

\section{N D E X}

qui

ordinem numerorum

Polypetalarum Disciflorarum Rehmannii sequitur.

141 Empleurum serrulatum Ait. 143 Agathosma cerefolium v. glabrata Sond.

145 Agathosma marifolia v. lanceolata Sond.

147 Barosma ovata v. vera Sz.

181 Rhus villosa L.

182 Rhus laevigata L.

184 Rhus villosa L.

187 Cassinopsis capensis Sond.

191 Rhamnus prinoides L'H.

192 Lauridia reticulata E. et Z.

198 Elaeodendron capense E. et Z.

211 Zanthoxylum Thunb. DC.?

212 (lausena inaequalis Bth.

232 Gymnosporia buxifolia v. laxiffora Sond.

238 Linum thesioides Bartl. 239

251 Oxalis corniculata L.
252 Geranium incanum L.

253 Monsonia ovata Cav.

254 Pelargonium alchemilloides n. dentatum Harv.

255 Pelargon. grossularioides v. anceps Harv.

256 Geranium canescens L'Her.

257 Pelargonium angulosum v. truncatum Szysz.

270 Vitis capensis v. Dregeana Kunth.

271 Vitis capensis v. Dregeana Kunth.

272 Rhus outeniquensis Szysz.

273 Vitis 'Thunbergii (E. et Z.).

274 Vitis capensis Th.

$2 \times 2$ Phylica trachyphylla Sond.

354 Cassine scandens v. latifolia Sond.

359 Ochna arborea Burch.

367 Pelargonium lobatum Willd. 
398 Putterlickia pyracant. (L.) 404 Agath. cerefolium v. glabrata Sond.

458 Zygopliyllum Morgsana L. 461 Monsonia ovata Cav.

462 Pelargon. lobatum Willd. 463 Pelargon. vitifolium Ait. 464 Pelargonium grossularioides v. auceps Harv.

478 Pelargonium longifolium v. ciliatum Harv.

486 Ekebergia Meyeri Pr.

487 Clausena inaequalis Bth. 555 Impatiens capensis 'Th. 563 Rhamnus prinoides L'H. 782 Phylica buxifolia L.

783 Phylica stipularis L.

807 Diosma succulenta Berg. 838 Oxalis polyphylla v. filifolia Sond.

839 Oxalis bifida Th.

840 Oxalis polyphylla v. filifolia Sond.

971 Hartogia capensis v. multiflora E. et Z.?

977 Phylica capitata Th.

1125 Pelargon. cucullatum Ait. 1126 p. p. Pelargonium astra. galifolium v. minor $\mathrm{H}$.

1125 p. p. Pelargonium astragalifolium v. minor $\mathrm{H}$.

1128 Pelargonium barbatum J. 1130 Pelargonium saniculaefolium Willd.

1131 Pelargonium myrrhifolium v. longicaule Harv.

1133 Monsonia speciosa L.

1139 Oxalis bifurca v. incana Harv.

1140 Oxalis purpurea Th.

1141 Rhus Dregeana Sond. 1142 Rhus angustifolia L.

1151 Diosma succulenta v. Lamarckiana Sond.
1152 Diosma succulenta v. Lamarckiana Sond.

1154 Agathosma imbric. v. acuminata Sond.

1155 Agatlosma imbric. v. acuminata Sond.

1156 Diosma vulgaris Schl.

1157

1245 Phylica plumosa Th.

1246 Phylica capitata Th.

1264 Lauridia reticul. E. et Z.

1265 Cassine Maurocenia L.

1267 Cassine capensis L.

1268 Gymnosporia laurina (E. et Z.).

1330 Oxalis purpurea $\mathrm{Th}$.

1331 Oxalis lanata L.

1332 Oxalis hirta L.

1333 Oxalis obtusa Jacq.

1335 a. Oxalis bifida Th.

1335 b. Oxalis bifurca Lodd.

1336

1337 Öxalis" bifurca v. incana Harv.

1339 Diosma virgata E. M.

1340 Agathosma imbric. v. reflexa Sond.

1341 Diosma succulenta v. Lamarckiana Sond.

1342 Adenandra cuspidatav. villosa Sond.

1343 Adenandra uniflora Willd.

1344 Diosma vulgaris v. longifolia Sond.

1345 Adenandra umbell. v. glandulosa B. et W.

1346 Rhus rosmarinifolia Vahl.

1347 Rhus Dregeana Sond.

1348 Rhus tomentosa L.

1349

1350 Rhus villosa v. glabrata Sond.

1351 Rhus discolor E. M. 1354 Oxalis corniculata L. 
1366 Pelargonium myrrbifolium v. longicaule Harv.

1367 Pelargonium myrrhifolium v. lacerum Harv.

1368 Erodium moschatum Willd. 1381 Linum thesioides Bartl.

1485 Oxalis flava v. Thunbergiana Sond.

1486 Oxalis tomentosa L.

1487 Oxalis hirta L.

1488

1489

$n " n$

1490

1491 Oxalis tenuifolia Jacq.

1492 Oxalis cernua Th.

1493 Oxalis sericea L.

1494

1495 Oxalis lanata L."

1496 Oxalis polyphylla v. filifolia Sond.

1497 a. Oxalis flava v. Thunbergiana Sond.

1497 b. Oxalis versicolor L.

1498 Oxalis bifurca $\mathrm{v}$, incana Harv.

1499 Oxalis bifida Th.

1500

1501

1502 Oxalis versicolor L.

1505 Oxalis lanata $\mathrm{I}$.

1506 Oxalis punctata L.

1507 Oxalis punctata L.

1508 Oxalis livida Jacq.

1509 Oxalis corniculata L.

1510 Oxalis variabilis v. nana siond.

1511 Oxalis purpurea Th.

\section{2}

1513

1526 Rhus pyroides Burch.

1527 Rhus mucronata v. Burmanni Sond.

1528 Rhus glauca Desf. 1529
1530 Rhus tomentosa L.

1531

1532 " angustifolia "L.

1533 Rhus stenophylla E. et Z.

$1534 "$ angustifolia L.

1536 " rosmarinifolia Vahl.

1545 Pelargon. grossularioides v. anceps Harv.

1551 Melia Azederach L.

1552 Melianthus major L.

1589 Oxalis sericea L.

1590 Oxalis hirta $\mathrm{L}$.

1591

1592 Oxolis bifurca v. incana Harv.

1593 Oxalis flava v. Thunbergiana Sond.

1600 Phylica ericoides L.

1603 Pelargon. capitatum Ait.

1609 Cassine Maurneenia L.

1610 Lauridia reticulata E. et Z. 1611

1612 Cassine barbata L.

1617 Gymnospuria laurina (E. et Z.).

1618 Lauridia reticulata E. et Z.

1619 Rhus pyroides Burch.

1620 Gymnosp. buxifolia v. venenata Sond.

1650 Gymnosp. buxifolia v. laxiflora Sond.

1651 Gymnosp. buxifolia v. venenata Sond.

1653 Vitis capensis Th.

1663 Scutia indica Brogn.

1678 Adenandra uniflora Willd.

1684 Linum Thunbergii E, et Z.

1691 Pelargonium myrrhifolium

v. longicaule Harv.

1695 Oxalis tenuifolia Jacq.

1696

1697 Oxalis bifurca Lod".

1698 Oxalis hirta L.

1699 
2018 Phylica buxifolia L.

2019

2020 Phylica eriophoros" Berg.

2021 (p. p.) Phylica stipular. L.

2021 (p. p.) Phylica plum. Th.

2146 Pelargon. betulinum Ait.

2147 Pelargon. capitatum Ait.

2148 Pelargonium myrrhifolium v. longicaule Harv.

2149 Pelargonium triste v. daucifolium Harv.

2150 Pelargonium triste v. filipendulifolium Harv.

2151 Pelargonium myrrhifolium v. fruticosum Harv.

2152 Geranium incanum L.

2173 Oxalis glabra v. pusilla S. 2174

" " " "

2176 Oxalis cernua Th.

2177 Oxalis commutata Sond.

2178 Oxalis versicolor $\mathrm{L}$.

2179 Oxalis obtusa Jacq.

2180 Zygophyll. sessilifolium L.

2181 Zygophyll. sessilifolium L.

2190 Linum thesioides Bartl.

2200 Gymnosp. buxifolia v. venenata Sond.

2202 Rhus discolor E. M.

2203 Rhus mucronuta v. Jacq. Sond.

2205 Rhus villosa v. glabrata Sond.

2206 Gymnosporia laurina (E. et Z.).

2207 Gymnosporia laurina (E. et Z.).

2208 Gymnosporia laurina (E. et Z).

2211 Diosma succulenta Berg.

2212 Diosma vulgaris Schl.

2213 Adenandra cuspidata v.glabra Sond.

2215 Agathosma rugosa v. pubescens Szysz.
2216 Diosma vulgaris Schl.

2217 Macrostylis villosa v. glabrata Sond.

2219 Agathosma rugosa v. lancifolia Szysz.

2234 Zygoph. flexuosum v. cuneatum Szysz.

2267 Rhus pyroides Burch.

2270 Gymnosporia laurina (E. et Z.).

2288 Hartogia capensis v. multiflora E. et Z.

2296 Phylica excelsa v. stricta Sond.

2317 Diosma vulgaris v. longifolia Sond.

2328 Anaphrenium argent. E. M. 2336 Dodonaea Thunbergian. E. et $\mathrm{Z}$.

2340 Hartogia capensis v. multiflora E. et Z.

2341 Gymnosporia laurina (E. et Z.).

2342 Anaphrenium argent. E. M. 2343 Pelargonium patulum v. tenuilobum Harv.

2345 Pelargonium rapaceum J.

2346

2347 Pelarỉonium scabrum Ait. 2380 Pelargonium crispum Ait.

2382 Rhus angustifolia L.

2383 Dodonaea Thunbergiana E. et Z.

2386 Phylica eriophoros v. Bergiana Sond.

2387 Phylica eriophoros v. Bergiana Sond.

2392 Gymnosporia laurina (E. et Z.).

2452 Pelargoniụm glaucum L'H.

2454 Pelargon. hispidum Willd.

2517 Rhus undulata Jacq.

2518 " incisa L.

2519 Rhus tomentosa $\mathrm{L}$. 
2524 Dodonaea Thunbergiana E. et Z.

2525 Dodonaea Thunbergiana E. et Z.

2533 Phylica stipularis L.

2534 Phylica excelsa v. papillosa Sond.

2535 Phylica excelsa v. papillosa Sond.

2540 Gymnosporia laurina (E. et Z.).

2541 Hartogia capensis v. lanceolata Sond.

2711 (p. p.) Phylica excelsa v. brevifolia Sond.

2711 (p. p.) Phylica excelsa v. laxa Sond.

2717 Barosma ovata v. cuneata Szysz.

2719 Rhus angustifolia L.

2720 Anaphrenium argenteum E. M.

2729 Pelargonium ovale Burm.

2772 Rhus lancea L.

2776 Pelargonium ceratophyilum L'H.

2814 Noltea africana Reich.

2817 Rhus angustifolia L.

2821 Rhus dissecta Thunb.

2823 Pelargonium ceratophyllum L'H.

2853 Pelargon. grossularioides v. anceps Harv.

2911 Rhus lancea L.

2912 Rhus undulata Jacq.

2944 Phylica villosa Th.

2951 Melianthus comosus Vahl.

2982 Tribulus terrestris v. desertorum Sond.

3003 Melianthus comosus Vahl. 3014 Elaeodendron glaucus Sz. 3050 Melianthus comosus Vahl. 3071 Rhus villosa L. 3075 Rhus lancea L.
3091 Dodonaea Thunberg. E. et $\mathrm{Z}$.

3095 Dodonaea Thunberg. E. et $\mathrm{Z}$.

3132 Zygophyl. microphyl. L. 3156 Zygophyll. glaucum E. M. 3180 Rhus viminalis v. pendulina Sond.

3223 Rhus ciliata Licht.

3336 Tribulus terrestris L.

3355 Zygophyl. microphyl. L. 3394 Zizyphus Spina-Christi Willd.

3433 Rhus ciliata Licht.

3442 Tribulus terrestris L.

3511 Monsonia Burkeana Pl.

3518 Rhus ciliata Licht.

3573 Zizyphus Spina - Christi Willd.

3586 Tribulus terrestris v. deserturum Sond.

3593 Rhus villosa $L$.

3595 Zizyphus Spina - Christi Willd.

3671 Monsonia biflora DC.

3795 Rhus erosa Thunb.

3832 Rhus erosa v. subintegra Szysz.

3841 Oxalis corniculata L.

3855 Monsonia speciosa L.

3869 Monsonia biflora DC.

3876 Oxalis commutata Sond.

3880 Rhus lancea L.

3897 Rhus erosa Thunb.

3947 Rhamnus prinoides L'H.

3981 Rhus erosa Thub.

3992 Geranium sericeum Harv. 4006 Rhus lancea L.

4078 Zizyphus helvola Sond.

4080 Gymnosporia polyacantha (Sond.).

4081 Gymnosp. buxifolia v. venenata Sond.

4083 Ochna arborea Burch. 
4084 Rhamnus prinoides L'H. 4094 Zanthoxylum capense H. 4099 Elaeodendron (?) Rehman. Szysz.

4194 Sphedamnocarp. pruriens f. major Szysz.

4195 Triaspis Rehmannii Szysz. 4233 Vitis erythrodes v. ferruginea Bak.

4341 Ochna Rehmannii Szysz.

4349 Monsonia ovata v. biflora Harv.

4352 Zizyphus mucronata v. pubescens Sond.

4354 Ximenia caffra Sond.

4571 Oxalis Mariae Szysz.

4572 Zizyphus mucronata v. glabrata Sond.

4573 Monsonia ovata v. biflora Harv.

4577 Vitis erythrodes v. ferruginea Bak.

4578 Vitis cirrhosa v. transvaalensis Szysz.

4582 Ochna arborea Burch. 4647 Helinus ovatus E. M.

4660 Zanthoxylum capense $H$. 4734 Sclerocarya caffra v. dentata Engl.

4735 Odina edulis Sond.

4736 Odina discolor Sond.

4737

4738

4739 Odina edulis v. glabrescens Engl.

4740 Rhus glaucovirens Engl. 4741 Rhus villosa $\mathrm{L}$.

4742 Rhus villosa v. gracilis Engl.

4743 Rhus Sonderi v. pilnsa Engl.

4744 Rhus Sonderi v. glaberrima Engl.

4745 Rhus coriacea Engl.
4747 Rhus lancea L.

4784 Vitis cuneifolia (E. et Z.). 4790 Sphedamnocarpus galphimiaefolius J.

4826 Tribulus terrestris v. hispidissimus Sond.

4851 Pelargon. Zeyheri Harv. 4852 Pelargon. Rehman. Szysz. 4869 Gymnosporia tenuispina (Sond.).

4879 Zizyphus mucron. Willd. 4881 Anaphrenium paniculosum Engl.

4882 Rhus gracillima Engl.

4883 Rhus viminalis Vahl.

4907 Tribulus terrestris v. hispidissimus Sond.

4913 Rhus incana Engl.

4944 Triaspis Rehmannii Szysz.

5005 Gymn. tenuispina (Szysz.).

5006 Gymnosp. buxifolia v. ge . nuina Sond.

5016 Monsonia biflora DC.

5030 Cardiospermum Halicacabum L.

5140 Sclerocarya caffra v. dentata Engl.

5141 Rhus lancea L.

5142 Rhus lanced L.

5143 Rhus Gueinzii Sond.

5154 Anaphrenium paniculosum Engl.

5204 Linum Thunbergii E. et Z.

5207 Tribulus terrestris L.

5325 Rhus incana Engl.

5326 Rhus villosa $\mathrm{L}$.

5327 Rhus Gueinzii Sond.

5328

5329 Rhus coriacea Engl.

5370 Rhus viminalis Vahl.

5374 Sclerocarya caffra Sond.

5458 Gymnosp. buxifolia v. genuina Sond.

5465 Ximenia caffra Sond. 
5479 Zizyphus helvola Sond.

5493 Sphedamnocarp. prur. J. 5495 Vitis erythrodes v. ferruginea Bak.

5498 Monsonia ovata v. lanci. folia Szysz.

5502 Helinus ovatus E. M.

5554 Rhus Gueinzii Sond.

5555 Rhus villosa L.

5556 Anaphren. paniculosum E.

5557 Rhus vilosissima Engl.

5558 Rhus acutidens Engl.

5559 Rhus transvaaleusis Engl.

5560 Rhus Rehmanniana Engl.

5561

5562 Vitis erythrodes v. ferruginea Bak.

5564 Vitis rhomboidea v. transvaalensis Szysz.

5565 Vitis Thunberg. (E. et Z.). 5566 Schmidelia magica Bak.? 5568 Rhus Rehmanniana Engl. 5569 Rhus laevigata L.

5571 Rlus viminalis Vahl.

5572 Rhus transvaalensis Engl. 5574 Rhus villosa $\mathrm{L}$.

\section{5}

6322 Monsonia Burkeana Pl.

6323 Monsonia ovata v. biflora $\mathrm{H}$.

6324 Pelargonium aconitifolium E. et Z.

6325 Pelargonium aconitifolium E. et Z.

6326 Pelargonium flabellifolium Harv.

6327 Pelargonium multicaule J. 6342 Linum Thunb. E. et Z. 6390 Sphedamnocarp. Rehmannii Szysz.

6391 Oxalis commutata Sond. 6392 Oxalis Smithii v. latifolia Sond.

6393 Oxalis caprina L. 6394 Oxalis luteola Jacq.
6395 Helinus ovatus E. M.

6396 Vitis cirrhosa (Th.).

6398 Vitis hypoleuca (Harv.). 6399 Impatiens capensis Th. 6400 6404 Greyia Radlkoferi Szysz. 6427 Gymnosp. acuminata (L.). 6457 Zizyphus helvola Sond. 6458 Zizyphus mucronata IVilld. 6459 Elaeodendron confertifolium v. leptocarpum S. 6463 Apodytes dimidiata E. M. 6465 Apodytes dimidiata E. M. 6497 Zanthoxylum capense $\mathrm{H}$. 6498 Zanthoxylum Thunb. DC.? 6499 Odina edulis Sond.

6501 Clausena inaequalis Bth. 6502 a.

6502 b. Poteroxylon utile f. "robusta Szysz.

6511 Gymnosp. acuminata (L.). 6512

6514 Gymnosporia nemorosa (E. et Z.).

6516 Gymnosp. luteola (Del.). 6517 Gymnosp. acuminata (L.). 6518 Gymnosp. huillensis (W.). 6523 Zizyphus mucronata Willd. 6571 Monsonia biflora DC.

6575 Rhus discolor E. M.

6597 Vitis erythrodes Fres.

6705 Rhus divaricata v. fulvescens Engl.

6819 Monsonia attenuata Harv. 6863 Rhus Sonderi v. pilosissima Engl.

6864 Rhus discolor v. paucinervia Engl.

6869 Melianthus Dregeana Sond. 6883 Rhus viminalis v. Gerrardi Engl.

6896 Rhus discolor v. brevifolia Engl.

6915 Monsonia attenuata Harv. 
6935 Geranium ornithopodum E. et Z.

6941 Rhus discolor v. brevifolia Engl.

6942 Rhus Zeyleri v. dentata Engl.

6943 Rhus viminalis v. Ger. E. 6960 Rhus villosa v. gracilis Engl.

6962 Impatiens capensis Th.

6963 Clausena inaequalis Bth. 6978 Vitis cuneifolia (E. et Z.). 6979 Vitis cuneifolia (E. et Z.). 6980 Vitis cuneifolia (E. et Z ). 70)9 Rhus villosa v. gracilis Engl.

7029 Hippobromus alatus E. et $\mathrm{Z}$.

7036 Helinus ovatus E. M.

7051 Rhus Rehmanniana Engl. 7052 Rhus refracta E. et Z.

7053 Rhus Rehmanniana Engl. 7054 Rhus refracta E. et Z.

7077 Greyia Sutherlandi Hook. 7098 Vitis natalitia Szysz.

7099 Vitis cirrhosa Th.

7103 Yelargon. alchemilloides v. dentatum Harv.

7136 Vitis natalitia Szysz.

7165 Clausena inaequalis Bth.

7178 Rhus excisa Tounb.

7218 Vitis 'Thunbergii (E. et Z.)

7220 Greyia Sutherlandi Hork.

724:3 Ochna atropurpurea v. natalitia Harv.

7268 Rhamnus prinoides L'H.

7279 Hippobromus alatus E. et $\mathrm{Z}$.

7281 Gymnosporia undata (Th.)

7351 Monsonia attenuata Harv.

7439 Rhus villosa v. gracilis Engi.

7489 Rhus villosa v. gracilis Engl.
7494 Clausena inaequalis Bth. 7503 Vitis rhomboidea (EM.). 7505 Helinus ovatus v. rotun difolius Sond.

7513 Zizyphus mucronata v. glabrata Sond.

7514 Zizyphus mucronata v. pubescens Sond.

7531 Zizyphus mucronata v. pu. bescens Sond.

7545 Zizyphus mucronata v. pubescens Sond.

7551 Erythroxylon emarginatum $\mathrm{S}$. et $\mathrm{T}$.

7572 Gymnosp. acuminata (L.).

7592 Oxalis pulchella v. glabrata Sond.

7593 Oxalis corniculata f. uniflora Szysz.

7690 Hippobromus alatus E. et $\mathrm{Z}$.

7691 Vitis cuneifolia (E. et 'Z.). 7692 Vitis cuneifolia (E. et Z.). 7699 Zizyphus mucronata v. glabrata Sond.

7707 Gymnosporia Rehmannii Szysz.

7758 Zizyphus mucronata v. glabrata Sond.

7765 Rhus dentata Thunb.

7767 Hippobrom. alatus E. et Z.

7771 Vitis capensis v. Dregeana Knth.

7784 Impatiens capensis Th.

7883 Putterlickia verrucosa S.

7785 Zizyphus mucronata v. gla. brata Sond.

7786 Ochna atropurpurea DC.

7912 Vitis capensis Th.

7991 Vitis natalitia Szysz.

8016 Scutia indica Brogn.

8042 Bersama lucens Szysz.

8048 Zizyphus mucronata v. glabrata Sond. 
8057 Scutia indica Brogn.

8072 Rhus crenata Thunb.

8105 Elaeodendron capense E. et $\mathrm{Z}$.

8106 Elaeodendron capense E. et $\mathrm{Z}$.

8115 Erythroxylon pictum EM.

8143 Turraen obtusifolia Hoch.

8149 Vitis cirrhosa Th.

8152 Vitis fragilis (EM.).

8170 Phylica paniculata Willd.

8173 Rhus dentata Thunb.

8174

8175 Rhus grandifolia "Engl.

8177 Rhus laevigata L.

8178 Rhus tridentata Sond.

8179 Odina edulis Sond.

8385 Vitis cuneifolia (E. et Z.).

8387 Rhus laevigata L.

8398 Pelargonium flabellifolium Harv.

8399 Pelargon. vitifolium Ait. 8400 Pelargon. alchemilloides v. dentatum Harv.

8429 Rhus crenata Thunb.

8430 Hippobrom. alatus E. et Z.

8513 Ekebergia capensis DC.

8515 Pelargonium aconitifolium E. et Z.

8525 Cardiospermum Halicacabum L.

8762 Vitis fragilis (E. M.).

8770 Sphedamnocarp. prur. (J.)

8773 Oxalis semiloba Sond.

8871 Monsonia ovata v. biflora Harv.

8872 Pelargon. grossularioides v. anceps Harv.

8873 Geranium ornithopodum E. et $\mathrm{Z}$.

8895 Elaeodendron capense E. et $\mathrm{Z}$.
8897 Elaeodendron capense E. et $\mathrm{Z}$.

8898 Elaeodendron capense E. et $\mathrm{Z}$.

8905 Ochna atropurpurea v. natalitia Harv.

8913 Turraea obtusifolia Hoch. 8956 Rhus natalensis Bernh.

8958 Apodytes dimidiata E. M. 8989 Putterlickia verrucosa S. 8990

8991

8992

9000 Scutia indica Brogn.

9001

9002 Scutia indica Brogn.

"016 Vitis rhomboidea (E. M.).

9017 Vitis rhomboidea (E. M.). 9018 Vitis Thunb. (E. et Z.). 9019 Vitis Thunb. (E. et Z.). 9020 Vitis Thunb. (E. et Z.). y021 Vitis capensis Th.

9022 Vitis cap. v. Dreg. Knth.

9030 Rhus laevigata L.

9033 Rhus laevigata L.

90:34 Rhus natalensis Bernh.

9037 Schmidelia natalensis S.

9038 Schmidelia Rehman. Sz. 9039 Schmidelia natalensis S.

$9040 \quad " \quad "$ $9041 n n " n$ $9042 \pi n$ 9043 Hippobrom. alat. E. et Z. 9044 Clausena inaequalis Bth. 9045 Zanthoxylum Thunb. DC.? 9046 Zanthoxylum cap. Harv. 9047 9048 9049 Melia" Azederach L. 9050 Ekebergia Meyeri Pr. 9057 Bersama lucens Szysz. 9064 Acridocarp. natal. Juss. 






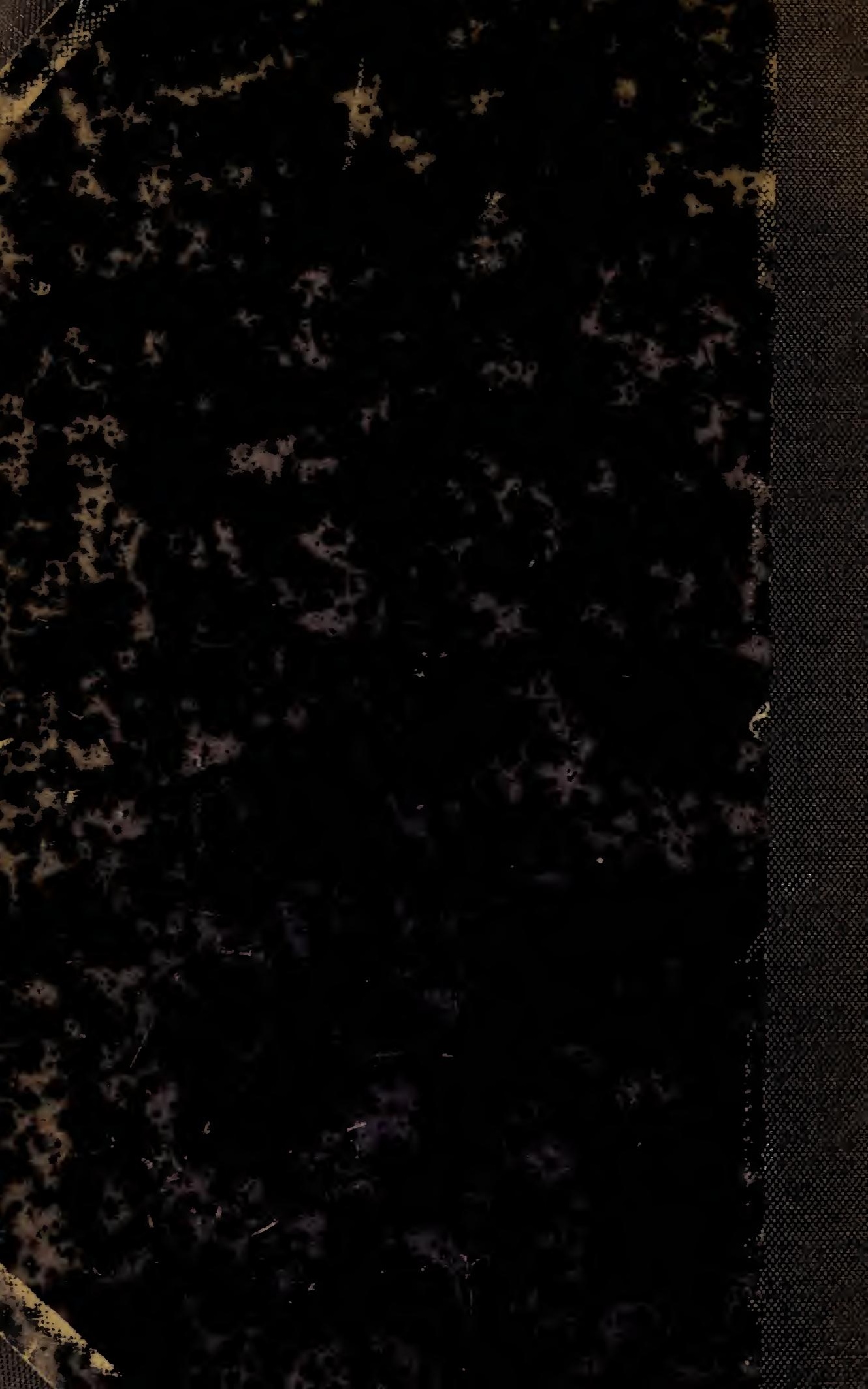

